ARTICLE

https://doi.org/10.1038/s41467-019-10741-9

\title{
Poly(ADP-ribose) polymerase-1 antagonizes DNA resection at double-strand breaks
}

Marie-Christine Caron 1,2,7, Ajit K. Sharma ${ }^{3,7}$, Julia O'Sullivan',2, Logan R. Myler ${ }^{4}$, Maria Tedim Ferreira ${ }^{2,5}$, Amélie Rodrigue ${ }^{1,2}$, Yan Coulombe ${ }^{1,2}$, Chantal Ethier ${ }^{2,5}$, Jean-Philippe Gagné ${ }^{2,5}$, Marie-France Langelier ${ }^{6}$, John M. Pascal ${ }^{6}$, Ilya J. Finkelstein (10 4, Michael J. Hendzel (10 ${ }^{3}$, Guy G. Poirier ${ }^{2,5}$ \& Jean-Yves Masson ${ }^{1,2}$

PARP-1 is rapidly recruited and activated by DNA double-strand breaks (DSBs). Upon activation, PARP-1 synthesizes a structurally complex polymer composed of ADP-ribose units that facilitates local chromatin relaxation and the recruitment of DNA repair factors. Here, we identify a function for PARP-1 in DNA DSB resection. Remarkably, inhibition of PARP-1 leads to hyperresected DNA DSBs. We show that loss of PARP-1 and hyperresection are associated with loss of Ku, 53BP1 and RIF1 resection inhibitors from the break site. DNA curtains analysis show that EXO1-mediated resection is blocked by PARP-1. Furthermore, PARP-1 abrogation leads to increased DNA resection tracks and an increase of homologous recombination in cellulo. Our results, therefore, place PARP-1 activation as a critical early event for DNA DSB repair activation and regulation of resection. Hence, our work has direct implications for the clinical use and effectiveness of PARP inhibition, which is prescribed for the treatment of various malignancies.

\footnotetext{
${ }^{1}$ Genome Stability Laboratory, CHU de Québec Research Center, HDQ Pavilion, Oncology Division, 9 McMahon, Québec City, QC G1R 3S3, Canada. ${ }^{2}$ Department of Molecular Biology, Medical Biochemistry and Pathology, Laval University Cancer Research Center, Québec City, QC G1V OA6, Canada. ${ }^{3}$ Department of Oncology, Faculty of Medicine and Dentistry, University of Alberta, 11560 University Avenue, Edmonton, AL T6G 1Z2, Canada. ${ }^{4}$ Department of Molecular Biosciences, University of Texas at Austin, Austin, TX 78712, USA. ${ }^{5}$ CHU de Québec Research Center, CHUL Pavilion, Oncology Division, 2705 Boulevard Laurier, Québec City, QC G1V 4G2, Canada. ${ }^{6}$ Biochemistry and Molecular Medicine, Université de Montréal, 2900 Boulevard Edouard-Montpetit, Pavillon Roger-Gaudry, Montréal, QC H3T 1J4, Canada. ${ }^{7}$ These authors contributed equally: Marie-Christine Caron, Ajit Sharma. Correspondence and requests for materials should be addressed to M.J.H. (email: mhendzel@ualberta.ca) or to G.G.P. (email: guy.poirier@crchudequebec.ulaval.ca) or to J.-Y.M. (email: Jean-Yves.Masson@crchudequebec.ulaval.ca)
} 


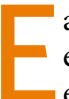
ach day, the eukaryotic genome is confronted with up to 50 endogenous DNA double-strand breaks (DSBs) ${ }^{1}$. These are extremely hazardous for a cell, because if left unrepaired, DSBs can have pathological consequences, such as cell death, or drive cells to genomic instability and tumor development. The cellular response to DNA damage involves an intricate network of enzymes responsible for sensing, signaling, and repairing damaged DNA, as well as the regulation of cell cycle checkpoints that collectively maintain genomic integrity ${ }^{2}$.

Poly(ADP-ribose) polymerase-1 (PARP-1) is an abundant and ubiquitous nuclear protein that uses $\mathrm{NAD}^{+}$to synthesize a multibranched polyanion composed of ADP-ribose moieties, giving rise to poly(ADP-ribose) (PAR), onto itself or a variety of target proteins. Protein ADP-ribosylation permits the transfer of the ADP-ribose moiety from $\mathrm{NAD}^{+}$to the side chain of several amino acids ${ }^{3-5}$. Predominant biological processes targeted by PARylation include RNA splicing, processing, and maturation, DNA replication, and transcription as well as the DNA damage response (DDR) ${ }^{3,6,7}$. PARP-1 acts as a highly sensitive sensor for DNA damage and rapidly produces PAR at newly generated DNA DSBs. This promotes local chromatin relaxation due to its negative charge ${ }^{8}$ and histone displacement ${ }^{9}$, as well as facilitating the recruitment of repair factors, such as MRE1 $11^{10}$. Several PARbinding modules orchestrate the relocation of DDR-associated factors in addition to the accumulation of intrinsically disordered proteins through an intracellular liquid demixing mechanism $^{11,12}$. PARP-1 is responsible for $80-90 \%$ of the global PAR synthesis following DNA strand breakage ${ }^{13}$. The dynamic turnover of PAR within seconds to minutes is executed by poly (ADP-ribose) glycohydrolase, the main PAR-degrading enzyme, which possesses both endoglycosidic and exoglycosidic activities, thereby enabling a new round of DNA damage signaling ${ }^{14}$. More recently, it has been shown that PARP-1 inhibition increases the speed of fork elongation and does not cause fork stalling, which is in contrast to the accepted model in which inhibitors of PARP induce fork stalling and collapse ${ }^{15}$. It was also recently shown that PARP-1 is a sensor of unligated Okazaki fragments during DNA replication ${ }^{16}$ and cells deficient in ribonucleotide excision repair are sensitized to PARP inhibition ${ }^{17}$.

PARP-1 is the best-characterized member of the diphtheria toxin-like ADP-ribosyl transferases (ARTDs) family of proteins. Among the 17 ARTDs members, only PARP-1, PARP-2, and PARP-3 are activated by DNA strand breaks ${ }^{18-20}$. De Murcia and colleagues provided the first evidence implicating PARP-1 in DNA repair by demonstrating that PARP-1-deficient mice are highly sensitive to $\gamma$-irradiation ${ }^{21}$. PARP-1 plays a critical role in DSB sensing and we have shown that PARP-1 recruitment and activation occur within $100 \mathrm{~ms}$ after introduction of DSBs. This makes PARP-1 activation one of the earliest and most critical events in the sensing of DSBs. Consistent with this, PARP-1 activity increases the rate of recruitment of the MRE11-RAD50NBS1 (MRN) complex ${ }^{10}$ and stimulates Ku binding in Dictyostelium $^{22}$. Structural analyses of PARP-1 have shown that PARP-1 binds DSBs. It does so through interactions with its zinc fingers and a WGR domain ${ }^{23-25}$.

In mammalian cells, most DSBs are repaired using long homologous sequences (homologous recombination (HR)), microhomology-mediated end joining, or no homology end joining (NHEJ). A key event that controls the DSB repair pathway choice is DNA end resection, which is characterized by $5^{\prime}$ to $3^{\prime}$ degradation of one strand at each side of the break. HR is initiated by CtBP-interacting protein (CtIP), a key molecular switch that controls DNA end resection and interacts with BRCA1. Although BRCA1 is a CtIP-interacting protein, there are conflicting reports on the roles of BRCA1 in DNA resection. While an early report found that disrupting the CtIP-BRCA1 interaction in DT40 cells diminished DNA-end resection ${ }^{26}$, a later report showed that CtIP mutated at Ser332, which is required for interaction with BRCA1, is competent for RPA and Rad51 assembly, indicating that resection is proficient in this background ${ }^{27}$. More recently, the CtIP-BRCA1 complex was found not to be essential for DNA end resection but rather modulated its speed ${ }^{28}$. The resection process is controlled by two core resection machineries in human cells: BLM-DNA2-RPA-MRN and EXO1-BLM-RPA-MRN ${ }^{29}$. DNA resection is also negatively regulated by the HELB helicase in an RPA-dependent manner ${ }^{30}$ and by 53BP1 and RIF1 proteins ${ }^{31,32}$.

Many years ago, we demonstrated that MRE11 and NBS1, which are core components of the early DSB sensing complex $M R N$, are recruited in a PARP-1-dependent manner to laserinduced DNA damage tracks. MRE11 was further shown to interact non-covalently with PAR via its intrinsically disordered glycine- and arginine-rich region, an interaction that modulates the resection functions of MRE11 $1^{10}$. We have previously shown that PARP-1 can interact with Ku70 and Ku80 33 and PARP-1 activity is necessary for $\mathrm{Ku}$ binding in Dictyostelium through PAR binding by $\mathrm{Ku} 70^{22}$. Importantly, PARP-1 activation precedes the recruitment of both the MRN and the Ku complex, previously recognized as the primary DSB sensors that recruit signaling proteins at DSB sites. Because the $\mathrm{Ku}$ complex and MRN bind PAR, PARylation may serve to guide and concentrate the $\mathrm{Ku}$ and MRN complexes at DSBs to facilitate their loading. As MRN is the initiator of DNA resection while the Ku complex inhibits end resection, we set out to determine whether PARP-1 could affect DNA resection.

The hypersensitivity of HR-deficient cancers to PARP inhibitors (PARPi) provided a conceptual basis for synthetic lethality. PARPi are currently being tested in over 200 clinical studies, with at least 50 trials in phase III (www.clinicaltrials.gov). Because of their specific mechanism of action, PARPi show a low toxicity profile ${ }^{34}$. PARPi has proven to be of significant clinical benefit, even for patients without HR deficiencies. Defining how PARP-1dependent DNA processing functions mechanistically will help identify genetic markers of sensitivity and resistance to guide PARPi therapy by identifying patients most likely to respond to either single agent or combination therapy through cytotoxic or radiation chemopotentiation ${ }^{35}$. Here, we identify PARP-1 as a critical regulator of DNA end resection of DSBs. We show that PARP-1 recruitment protects DNA ends from nucleolytic degradation and inhibition of PARP-1 leads to hyper-resected DNA double-strand breaks. Our data provide an alternative mechanism by which PARPi function in the presence of irradiation (IR).

\section{Results}

Recruitment of PARP-1 and PARP-2 at laser-induced DNA breaks. We initially scrutinized the recruitment kinetics of PARP1 to laser-induced DNA damage. Consistent with previous findings ${ }^{36,37}$, we observed that PARP-1 is recruited rapidly to laser-induced DNA damage tracks within milliseconds (Supplementary Fig. 1). The dynamics of PARP-1 recruitment under normal conditions was compared with the dynamics observed under PARP inhibition with BMN 673 (Talazoparib). The initial rapid accumulation of PARP-1 at sites of damage was followed by a steady reduction over the next $10 \mathrm{~min}$, while in the presence of BMN 673, PARP-1 is lost from the damage site more slowly, possibly due to trapping at $\mathrm{DSBs}^{38}$. Similar to PARP-1, PARP-2 retention at laser-induced DNA lesions is normally transient, with a slow decline after an initial maxima at approximately 2 min post damage. However, in cells exposed to BMN 673, an initial rapid accumulation is observed, but rather than decline, there persists a slowly increasing accumulation of PARP-2 over the $10 \mathrm{~min}$ experiment (Supplementary Fig. 2). Because the 
ultrafast recruitment of PARP-1 at DNA lesions precedes and primes the accumulation of other DNA damage repair factors, including the MRN complex, and because PARylation is a determining factor for their local accumulation, we reasoned that PARylation-dependent events might also affect DSB repair pathway choice through DNA end resection.

Effect of PARPi on DNA end resection. We used two different methods to determine whether PARP-1 itself or its catalytic activation influences DNA resection. First, we used a bromodeoxyuridine (BrdU)-based assay for visualizing the singlestranded DNA (ssDNA) product of resection. Second, we measured the accumulation of replication protein A (RPA). RPA is an essential trimeric protein complex that binds to ssDNA in eukaryotic cells. It is recruited to sites of DNA damage when regions of ssDNA are exposed. Hence, it serves as a readout for resection and for ongoing HR. Thus the amount of RPA that accumulates at each site should reflect the amount of ssDNA. Remarkably, both PARP-1 inhibition by BMN 673 or small interfering RNA (siRNA)-mediated PARP-1 silencing led to a substantial increase in the generation of ssDNA as measured by BrdU intensity in U2OS or HeLa cells (Fig. 1a, c). This was further confirmed using CRISPR/Cas9-mediated knockout of PARP-1 in 293T cells (Supplementary Fig. 3A, B). We quantified this data by measuring the total amount of BrdU (or RPA) in each focus. The summed intensity values for each individual focus reveals a greater than two-fold increase in the average amount of ssDNA generated per DSB (focus) when PARP-1 is inhibited by BMN 673 or silenced by siRNA. Consistent with an accumulation of RPA foci following pharmacological inhibition of PARP-1 or PARP-1 knockdown (Fig. 1b, d, Supplementary Fig. 3C), we observed an overall increase of chromatin-bound and phosphorylated RPA (Fig. 1e). Microirradiation experiments also showed enhanced recruitment of GFP-RPA1, GFP-RPA2, or GFP-RPA3 in S-phase cells following pharmacological inhibition of PARP-1 (Fig. 1f, Supplementary Fig. 3E, F). It is well known that the activation of the ATR kinase following perturbations in S-phase relies on a complex mechanism involving ATR recruitment to RPA-coated ssDNA. Consistent with an increase in RPA recruitment in PARP-1 knockdown cells, the activation of ATR was enhanced as judged by using anti-ATR Thr-1989 as a proximal marker of ATR activation ${ }^{39}$ (Supplementary Fig. 3G).

To rule out the possibility that PARP-1 plays an indirect role that promotes the accumulation of ssDNA, we repeated the BrdU-based assay with a knockdown of CtIP, which is expected to suppress DNA resection at $\mathrm{DSBs}^{40}$. The simultaneous knockdown of PARP-1 and CtIP completely suppressed the accumulation of ssDNA, implying that knockdown of PARP-1 affects the DSB resection process and does not promote a resectionindependent accumulation of ssDNA (Fig. 1g, Supplementary Fig. 4A, B). CtIP foci formation was also increased in PARP-1 knockdown cells (Supplementary Fig. 4C, D). The accumulation of ssDNA was not observed in DNA Ligase IV knockdown cells, suggesting that cells compromised in later stages of NHEJ do not share this phenotype (Supplementary Fig. 4E). Since we are monitoring DNA resection products $3 \mathrm{~h}$ after IR, we ascertained that the above results were not a consequence of an accumulation of cells in S/G2 (Supplementary Fig. 5A, B). In addition to the intensity, the number of BrdU or RPA foci per nucleus were increased in BMN 673-treated U2OS cells or PARP-1-silenced HeLa cells compared to controls (Supplementary Fig. 5C-F). Treatment with another PARPi, Veliparib, caused also an enhancement of BrdU or RPA intensity per nucleus (Supplementary Fig. 6A, B).
To quantify ssDNA at the sites of DSBs, we used the ER-AsiSI system in which the restriction enzyme AsiSI is fused to the estrogen receptor hormone-binding domain. Upon treatment with 4-hydroxytamoxifen (4-OHT), the AsiSI nuclease translocates to the nucleus and generates up to 150 DSBs at sequencespecific sites ${ }^{41,42}$. In this system, the presence of DSB resection will lead to the generation of ssDNA that cannot be cleaved by the duplex DNA-specific endonuclease BsrGI before PCR. In the absence of DNA resection, the remaining double-stranded DNA (dsDNA) will be cleaved, therefore yielding no PCR products (Fig. 2a). Thus the system can be used to distinguish between ssDNA and dsDNA. Interestingly, PARP-1 depletion or inhibition by BMN 673 (Fig. 2b) led to a $\sim 30 \%$ increase in DNA resection compared to the control at two different sites (Fig. 2c). Similarly, PARP-1 inhibition led to a 3-6-fold increase in bound RPA2 to processed DSBs (Fig. 2d). Altogether, these results show that PARP-1 limits DNA processing in cellulo.

PARP-1 knockdown cells show decreased 53BP1 and RIF1 foci. The mechanism underlying PARP-1-regulated DNA resection was investigated further. We monitored the accumulation of the resection inhibitors 53BP1 and RIF1 in G1 cells (Fig. 3a, b) depleted of PARP-1. Interestingly, PARP-1 inhibition led to a decrease of 53BP1 and RIF1 foci following etoposide treatment (average number of foci $=46$ in the control and 28 in PARP-1 knockdown for 53BP1, and average number of foci $=27$ in the control and 11 in PARP-1 knockdown for RIF1). These results were corroborated by chromatin immunoprecipitation (ChIP) analysis using cells stably expressing mCherry-LacI-FokI at an integrated reporter transgene (U2OS-DSB-reporter system (Fig. 3c)), which showed a drastic reduction of 53BP1 (Fig. 3d) or RIF1 accumulation (Fig. 3e) on two different DSBs in PARP-1 knockdown or BMN 673-treated cells.

PARP-1 blocks DNA resection by MRN-RPA-BLM-EXO1DNA2. The above data suggest that PARP-1 may be able to directly suppress the activity of DNA resection enzymes. We further examined whether purified PARP proteins (Fig. 4a) could block DNA resection in vitro. We monitored DNA resection of a $3^{\prime}$-end-labeled dsDNA $(2.7 \mathrm{~kb})$ via the two main DNA resection machineries MRN-RPA-BLM-EXO1 or MRN-RPA-BLM-DNA2. In the absence of PARPs, the MRN-RPA-BLM-EXO1 assembly resected $\sim 75 \%$ of the $2.7 \mathrm{~kb}$ substrate (Fig. $4 \mathrm{~b}$ ). When the reaction was supplemented with PARP-1, a concentration-dependent inhibition was observed. At $50 \mathrm{nM}$ PARP-1, only $10 \%$ of the DNA could be resected within the $60 \mathrm{~min}$ incubation time, without NAD or at a NAD concentration that still supports DNA binding. As specificity controls, we also performed similar reactions with PARP-2 and PARP-3. Importantly, PARP-2 and PARP-3 enzymes did not inhibit MRN-RPA-BLM-EXO1-mediated DNA degradation. PARP-1 also blocked the MRN-RPABLM-DNA2 complex and PARP-1-mediated inhibition was retained with the catalytic mutant $\mathrm{E} 988 \mathrm{~K}$ but abolished when a PARP-1 fragment devoid of its zinc fingers (PARP-1 216-1014) was used (Fig. 4c, Supplementary Fig. 6C). PARP-1 216-1014 has been reported to have a severely decreased affinity (250-fold less) for DNA lesions compared to wild-type (WT) PARP-1 ${ }^{23}$. PARP-1 blocked the resection complexes at 5 and $30 \mu \mathrm{M}$ concentration of $\mathrm{NAD}^{+}$, where PARP-1 undergoes only moderate PAR automodification (Supplementary Fig. 6D, E) and remains bound to DNA (Fig. 4b-d, Supplementary Fig. 6F, G). In contrast, the higher $\mathrm{NAD}^{+}$concentration of $250 \mu \mathrm{M} \mathrm{NAD}{ }^{+}$, where PAR automodification releases PARP-1 from DNA, prevented PARP-1 inhibition of resection (Fig. 4e, Supplementary Fig. 6F, G). Since even low levels of PAR alone could sequester protein components 
a

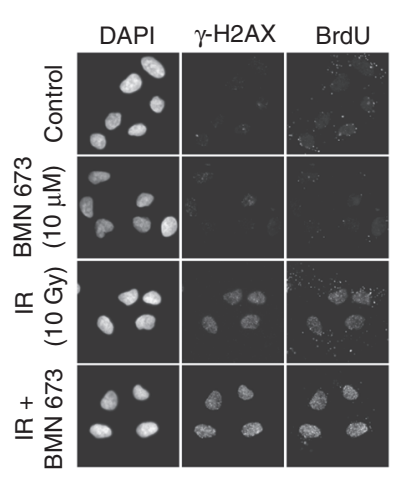

C

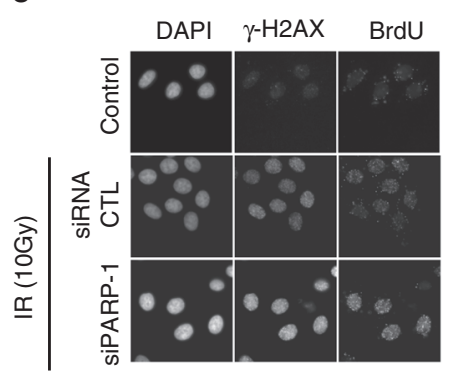

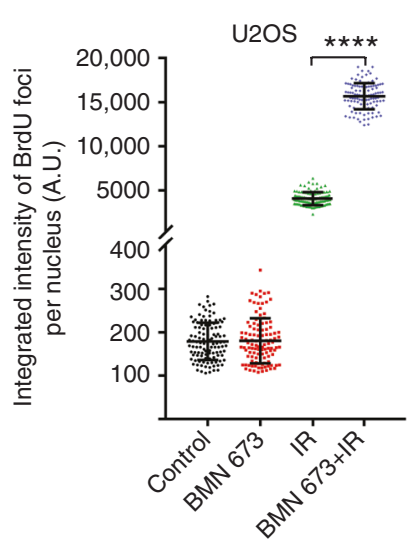

b

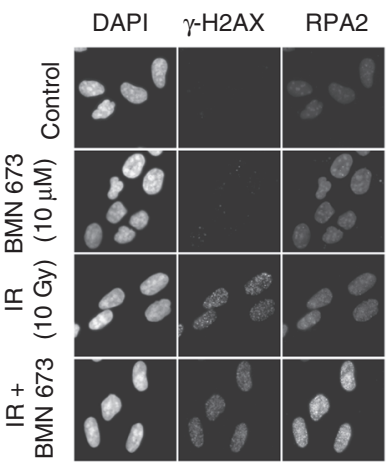

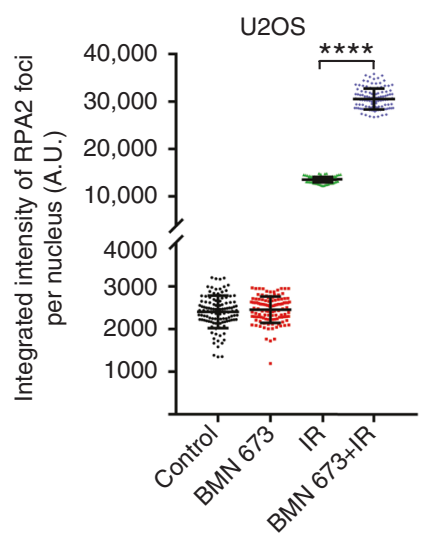

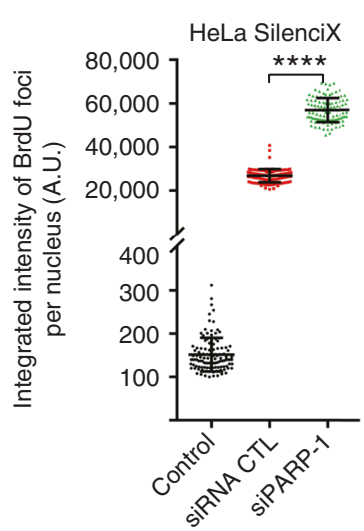

d

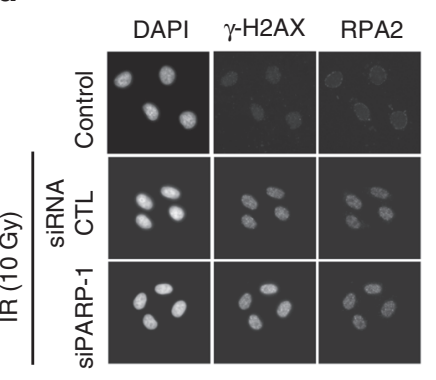

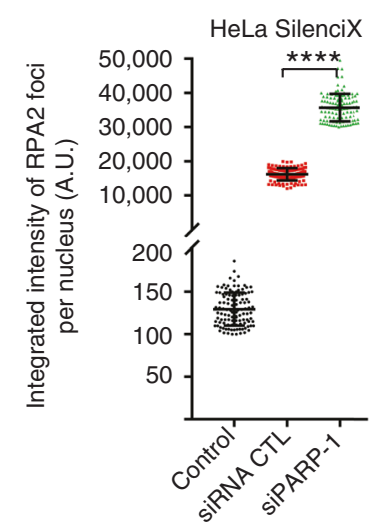

e

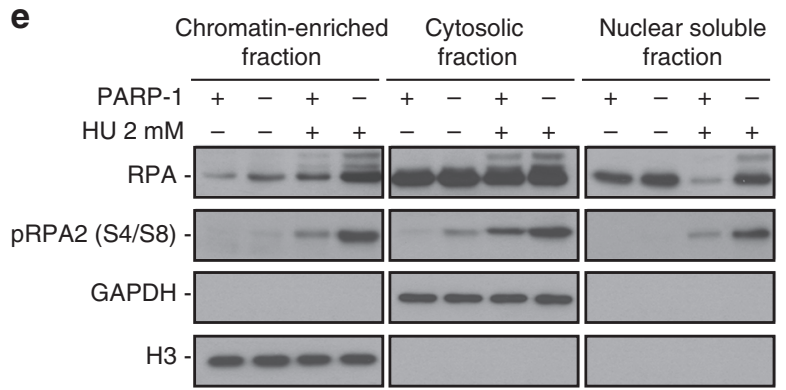

$\mathbf{f}$

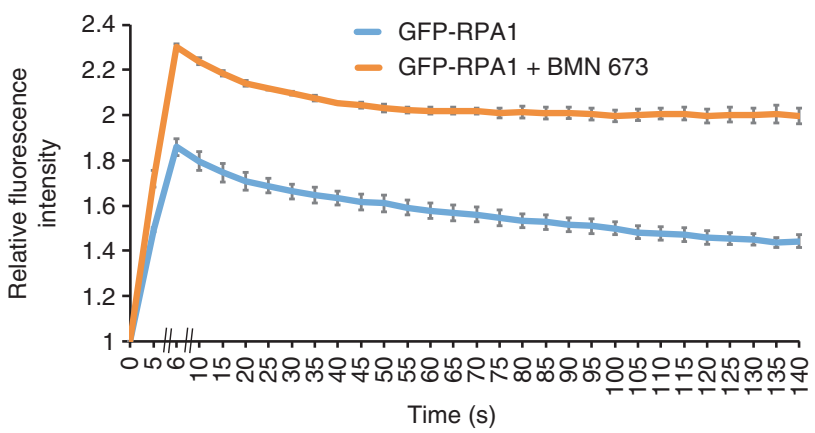

g
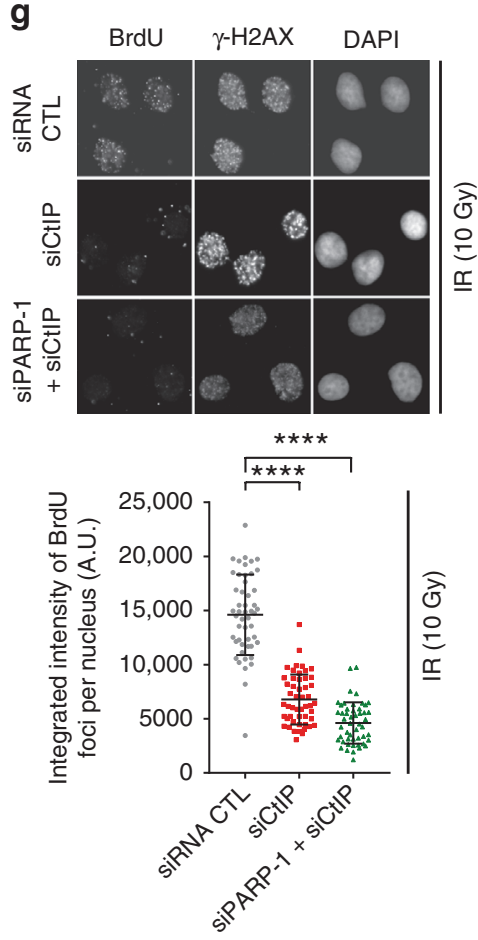

of the resection reaction leading to suppression of DNA resection, we performed reactions with protein-free PAR (Fig. 4f). This failed to inhibit DNA resection. These results show that PARP-1 but not PARP-2, PARP-3, or PAR can robustly inhibit DNA resection through a direct DNA-binding mechanism.

We used a high-throughput single-molecule DNA curtain assay to directly observe how PARP-1 inhibits EXO1 processing of substrate DNA. Arrays of DNA molecules $(48.5 \mathrm{~kb}$, derived from $\lambda$-phage) were assembled on the surface of a microfluidic flowcell coated with a surface-passivating fluid lipid bilayer ${ }^{43}$. The DNA substrate was linked to the bilayer via a biotin-streptavidin linkage. Fluorescently labeled PARP-1 was injected into the flowcell and visualized using total internal reflection fluorescence microscopy on thousands of DNA molecules for high-throughput 
Fig. 1 Poly(ADP-ribose) polymerase-1 (PARP-1) regulates DNA end resection and chromatin accumulation of replication protein A (RPA). U2OS cells mock treated, treated with $B M N 673$, irradiated $(10 \mathrm{~Gy})$, or irradiated (10 Gy) in combination with BMN 673 were subjected to immunofluorescence against $\gamma-\mathrm{H} 2 \mathrm{AX}$, bromodeoxyuridine (BrdU) (a), or RPA2 foci formation (b). HeLa SilenciX cells underexpressing PARP-1 by siRNA-mediated gene knockdown were subjected to immunofluorescence against $\gamma-\mathrm{H} 2 \mathrm{AX}, \mathrm{BrdU}$ (c) or RPA2 foci formation after irradiation (10 Gy, $3 \mathrm{~h}$ release) (d). In panels a-d, the data show the mean \pm s.d. (Mann-Whitney $U$ test). e HEK293T wild type or CRISPR-PARP-1 were treated with 2 mM hydroxyurea for 16 h, fractionated into chromatin enriched, nuclear soluble and cytoplasmic fractions. f GFP-RPA1 recruitment in the absence (blue line) or presence of BMN 673 (orange line). The peak of intensity was at $6 \mathrm{~s}$. Data show the mean \pm s.e.m. $\mathbf{g}$ knockdown of CtIP suppresses the accumulation of single-stranded DNA in HeLa SilenciX PARP-1 knockdown cells after irradiation (10 Gy, $3 \mathrm{~h}$ release). The data show the mean \pm s.d. ${ }^{\star \star \star \star} p \leq 0.0001$ (Mann-Whitney $U$ test). Source data are provided as a source data file

a

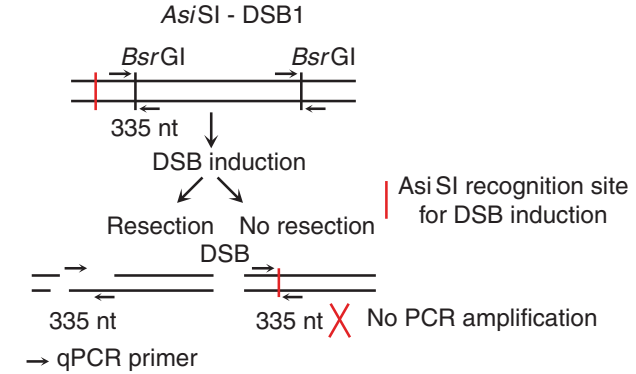

b

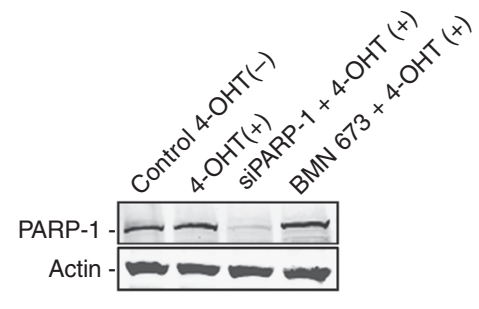

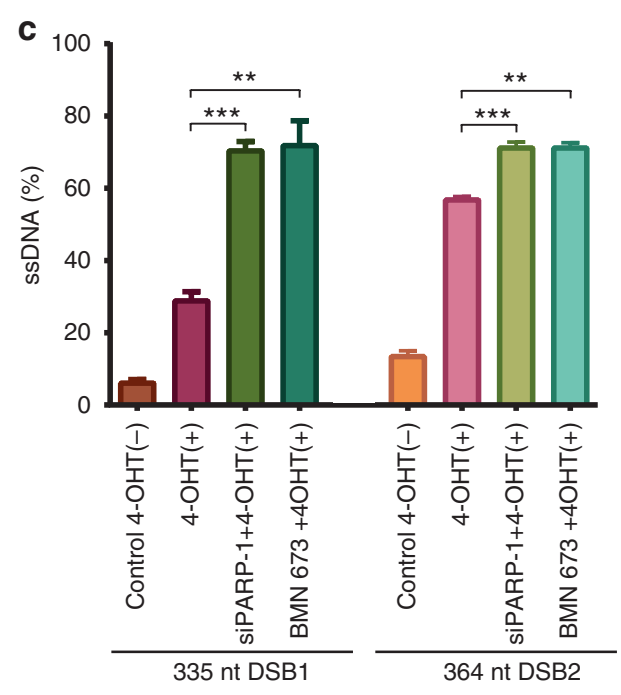

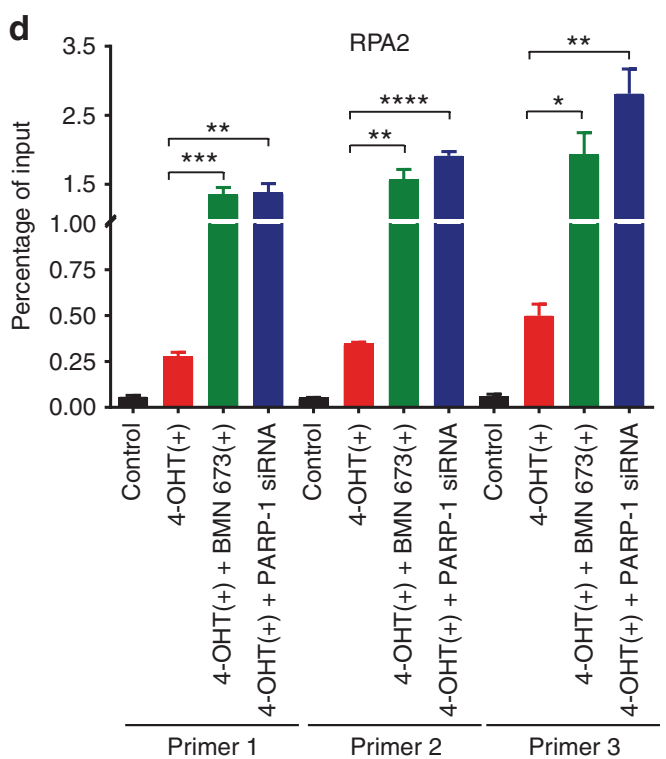

Fig. 2 Measurement of double-strand break (DSB) resection in ER-AsiSI U2OS cells knocked down for poly(ADP-ribose) polymerase-1 (PARP-1) or treated with a PARP inhibitor. a Quantitative polymerase chain reaction ( $\mathrm{QPCR}$ ) primers and probes for measurement of DSB resection at two AsiSI sites. $\mathbf{b}$ ER-AsiSI U2OS cells were knocked down for PARP-1 or treated with BMN 673 for $1 \mathrm{~h}$, followed by induction with $300 \mathrm{nM}$ 4-hydroxytamoxifen for $3 \mathrm{~h}$. c Quantitation of the percentage of DNA resection. Error bars represent mean \pm s.e.m. ${ }^{\star \star} p \leq 0.01,{ }^{\star \star \star} p \leq 0.001$ (Mann-Whitney $U$ test). $\mathbf{d}$ Chromatin immunoprecipitation-qPCR was performed with antibody against RPA2 in the ER-AsiSI U2OS cells. DSBs were induced at 48-72 $\mathrm{h}$ after siRNA transfection or after $1 \mathrm{~h}$ BMN 673 treatment. Immunoprecipitated chromatin samples were analyzed by qPCR using specific primer pairs located at Chr 1 :

chr1_89231183, Chr 6: chr6_90404906, and Chr 21: distal region of chr21_21292316. Error bars represent mean \pm s.e.m. ${ }^{\star} p \leq 0.05,{ }^{\star \star} p \leq 0.01$, ${ }^{\star \star \star} p \leq 0.001$, ${ }^{\star \star \star \star} p \leq 0.0001$, (Mann-Whitney $U$ test). Source data are provided as a source data file

data collection and analysis (Fig. 5a). Nearly all PARP-1 molecules were bound to the end of the DNA. This conclusion is based on the observation that turning off buffer flow led to the coordinated retraction of both the DNA and associated PARP-1 molecules to the diffusion barrier (Fig. 5b, c). Most PARP-1 molecules localized to the DNA end via binding internally and sliding along the DNA in the direction of buffer flow $(88 \%$ of PARP-1 molecules, $N=109 / 124)$. This indicates that PARP-1 is able to diffuse along the DNA and one-dimensional (1D) diffusion may represent a mechanism by which PARP-1 quickly associates with DNA ends or other DNA lesions. In conditions where PARP-1 was pre-bound to the end of DNA molecules (Fig. 5d, magenta in right panel), a second PARP-1 molecule (Fig. 5d, green in right panel) bound upstream and slid along the DNA to co-localize at the DNA end. Consequently, the red and green traces can be seen to merge in the kymograph (Fig. 5d). Pre-bound PARP-1 blocked DNA resection by preventing EXO1 loading onto the DNA end (Fig. 5e-g). This suggests that PARP-1 may physically occlude the end of DNA. In contrast, end-bound PARP-2 did not block EXO1 loading and DNA resection. 

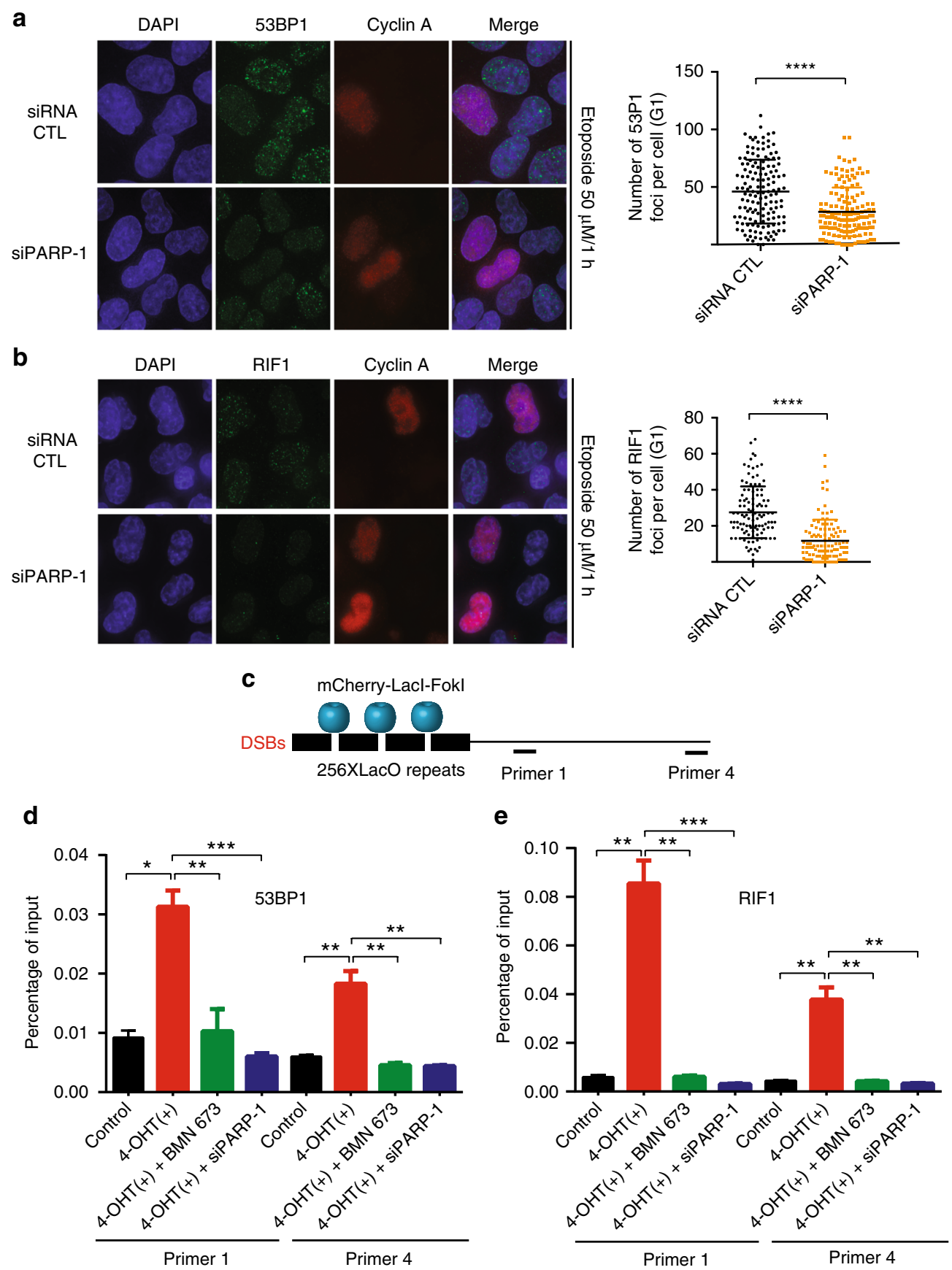

Fig. 3 Poly(ADP-ribose) polymerase-1 (PARP-1) inhibition reduces the accumulation of 53BP1 and RIF1 foci. a Etoposide-treated HeLa cells were either transfected with an small interfering RNA (siRNA) control or PARP-1 siRNA and subjected to immunofluorescence staining against 53BP1 or Cyclin A. The mean foci count was 46.05 for siRNA CTL and 28.42 for siPARP-1. Data show the mean \pm s.d. ${ }^{\star \star \star \star} p \leq 0.0001$ (Mann-Whitney $U$ test). $\mathbf{b}$ Etoposide-treated HeLa cells were either transfected with a control siRNA or PARP-1 siRNA and subjected to immunofluorescence staining against RIF1 or Cyclin A. The mean foci count was 27.52 for siRNA CTL and 11.68 for siPARP-1. Data show the mean \pm s.d. ${ }^{\star \star \star \star} p \leq 0.0001$ (Mann-Whitney $U$ test). c Cartoon of the U2OSDSB-reporter with inducible DSB generation by mCherry-Lacl-Fok1. Chromatin immunoprecipitation (ChIP)-quantitative polymerase chain reaction ( $q P C R$ ) primer sets are shown as p1-p4. ChIP of 53BP1 (d) or RIF1 (e) from the fixed chromatin of U2OS-DSB reporter cells stably expressing mCherry-Lacl-Fok1 construct and treated with both $0.5 \mathrm{mM}$ Shield-1 (1:500 dilution) and $1 \mathrm{mM}$ 4-hydroxytamoxifen (4-OHT) for $1 \mathrm{~h}$ in order to generate DSBs. The ChIP-qPCR primer sets are labeled as primer 1 and primer 4. Data in d-e show the mean \pm s.e.m. ${ }^{\star} p \leq 0.05,{ }^{\star \star} p \leq 0.01,{ }^{\star \star \star} p \leq 0.001($ Mann-Whitney $U$ test). Source data are provided as a source data file

Consistent with this, microirradiation experiments showed enhanced accumulation of GFP-EXO1 in PARP-1-deficient cells at laser-induced DNA damage sites (Fig. 5h) and phosphorylated EXO1 accumulated more in the absence of PARP-1 (Supplementary Fig. 7A). Altogether, our results show that PARP-1 counteracts DNA resection in vitro, likely by occluding the free DNA ends from the EXO1 or DNA2 resection machineries.

PARP-1 is required for efficient loading of the $\mathrm{Ku}$ complex. Another mechanism whereby PARP-1 could inhibit end resection 
a

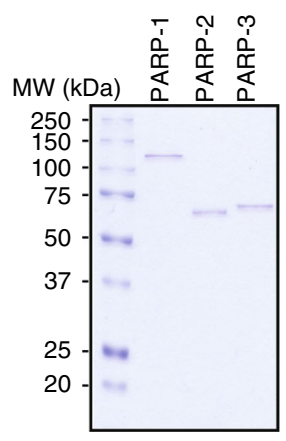

b

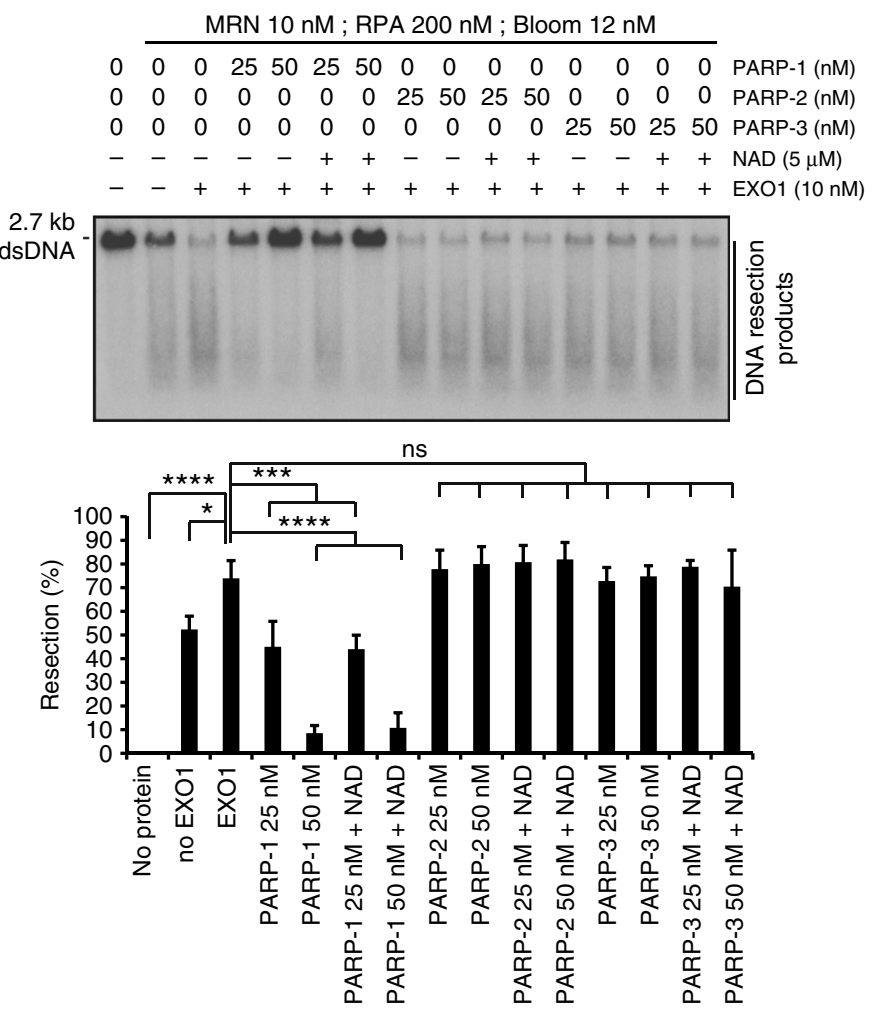

C MRN $10 \mathrm{nM}$; RPA $100 \mathrm{nM}$;
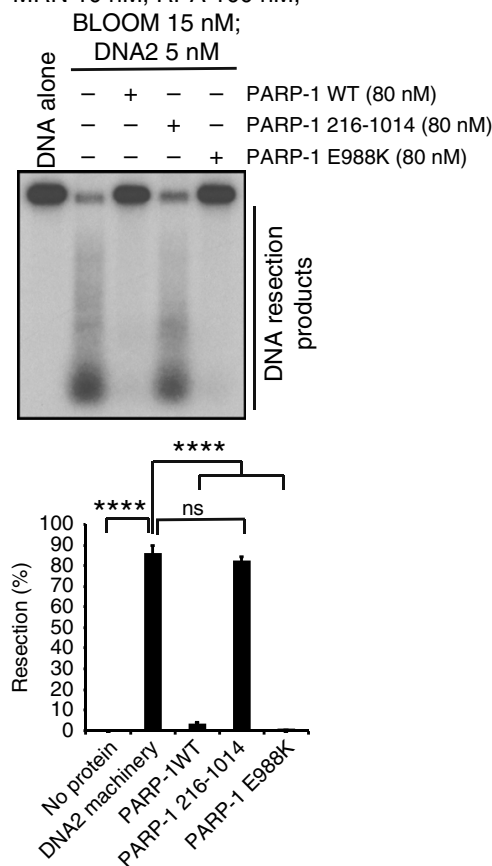

d

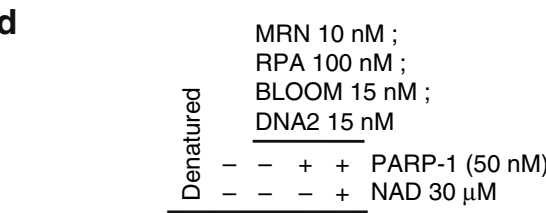

MRN $10 \mathrm{nM}$; RPA $100 \mathrm{nM}$; BLOOM $15 \mathrm{nM}$; DNA2 $15 \mathrm{nM}$ - $40804080-\ldots \ldots$ - _ PARP-1 (nM) _ _ _ _ - 40804080 - $~-~-~ P A R P-2(n M)$ - - _ - _ - - 40804080 $---++-\ldots++-++$ PARP-3 (nM) $2.7 \mathrm{~kb}$ dsDNA
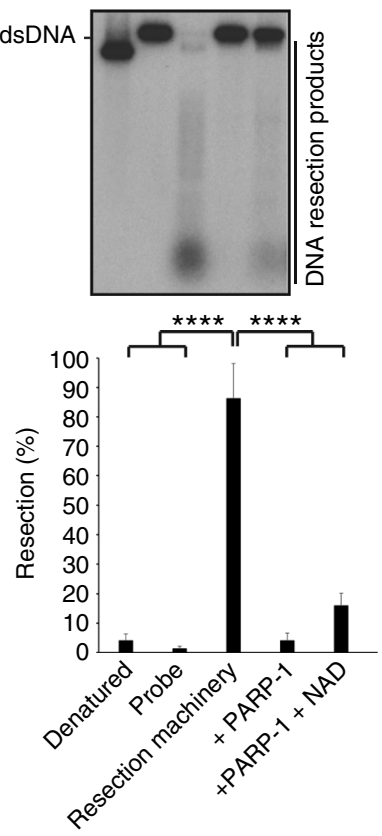
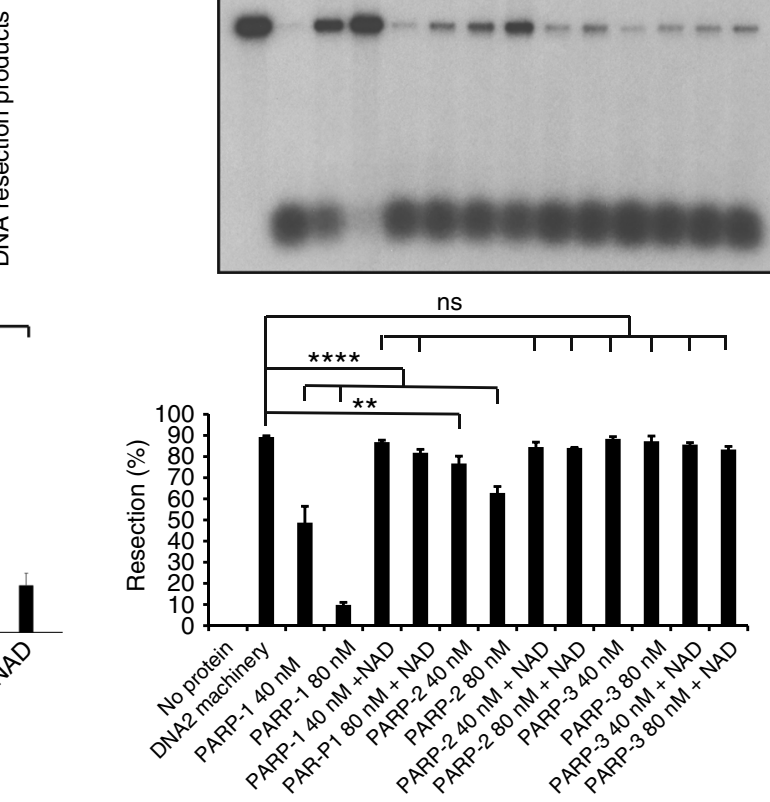

f

MRN $10 \mathrm{nM}$; RPA $100 \mathrm{nM}$; Bloom $15 \mathrm{nM}$; DNA2 $15 \mathrm{nM}$

-++-+ PARP-1

$-\quad-\quad+\quad+$ PAR $250 \mu \mathrm{M}$
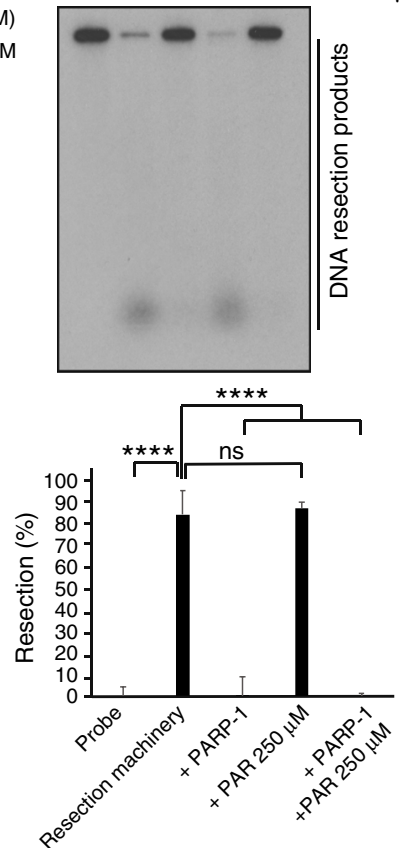

is through regulation of the association of the $\mathrm{Ku}$ complex with DNA ends. Consequently, we performed laser microirradiation experiments to determine whether Ku80-GFP recruitment to sites of DNA damage is sensitive to PARPis (Fig. 6a, b). We found that the recruitment of Ku80-GFP was almost completely inhibited in cells treated with PARPi but robustly recruited in control cells. We next tested PARP-1-deficient cells and found that they also fail to efficiently recruit Ku80-GFP to sites of DNA damage (Fig. 6c, d). In order to determine the influence of PARP activity on the association of Ku80 with DNA ends, we performed ChIP experiments in the presence or absence of PARP-1 or PARPi (Fig. 6e) and in the presence of RNAse to avoid indirect binding through 
Fig. 4 Poly(ADP-ribose) polymerase-1 (PARP-1) limits DNA end resection in vitro. a Sodium dodecyl sulfate-polyacrylamide gel electrophoresis of purified human PARP-1, PARP-2, and PARP-3. b The indicated PARP proteins were incubated with the MRN-RPA-BLM-EXO1 machinery in the absence or presence of NAD $(5 \mu \mathrm{M})$. The resection products were detected by autoradiography after agarose gel electrophoresis. Bottom: quantification of the results.

c The PARP-1 DNA-binding mutant PARP-1 216-1014 fails to inhibit DNA resection. Bottom: quantification of the results. $\mathbf{d}$ DNA end resection by the MRNRPA-BLM-DNA2 machinery is decreased in the absence or presence of NAD $(30 \mu \mathrm{M})$. e The indicated PARP proteins were incubated with the MRN-BLMRPA-DNA2 machinery in the absence or presence of NAD $(250 \mu \mathrm{M})$. $\mathbf{f}$ The addition of purified PAR $(250 \mu \mathrm{M})$ alone does not block DNA resection by MRN-BLM-RPA-DNA2. PARP-1 was used at $87 \mathrm{nM}$. Error bars in b-f indicate s.d. from three independent experiments. ${ }^{\star} p \leq 0.05,{ }^{\star \star} p \leq 0.01,{ }^{\star \star \star} p \leq 0.001$, ${ }^{\star \star \star \star} p \leq 0.0001$ (ordinary one-way analysis of variance). Source data are provided as a source data file

a

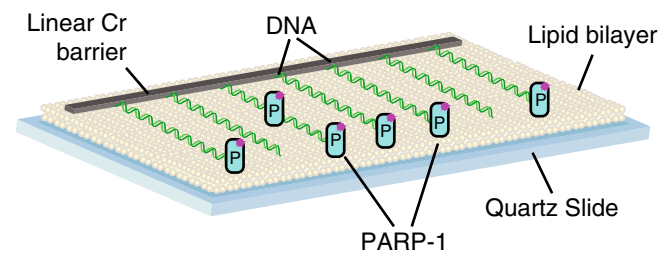

b
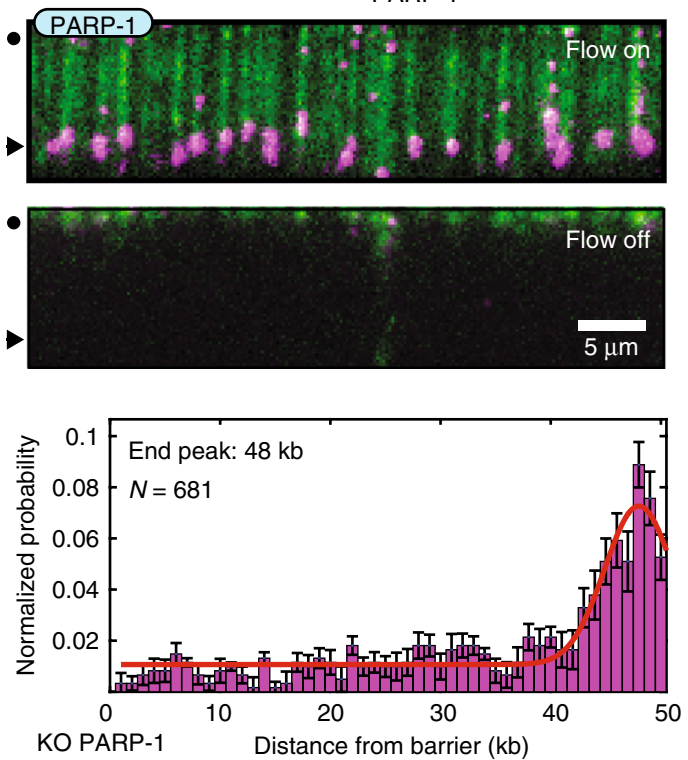

h

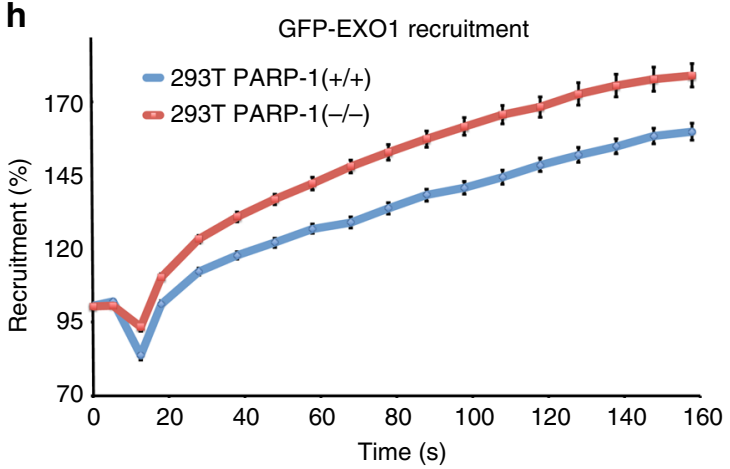

d

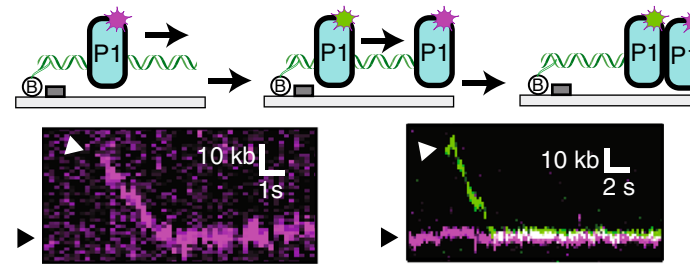

e EXO1 injection

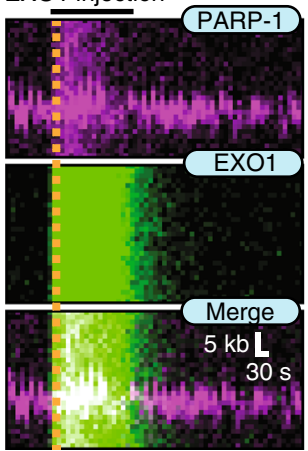

EXO1 injection

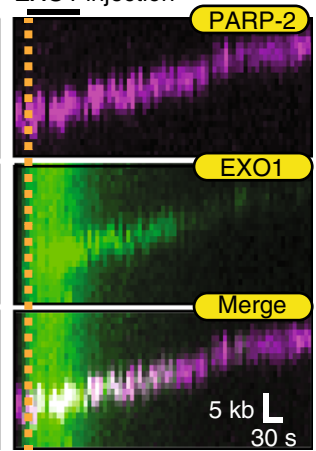

f

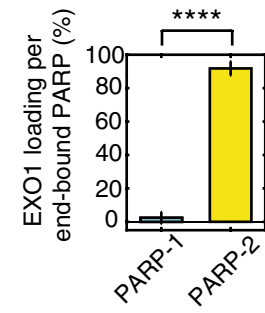

g /Janara
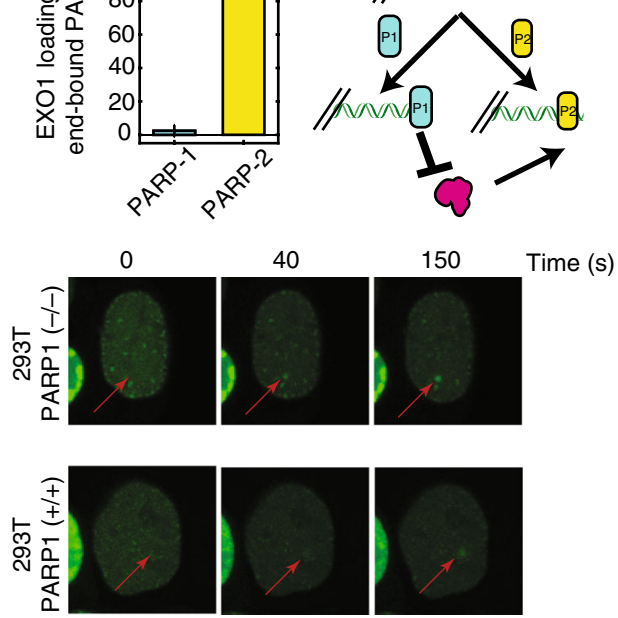

Fig. 5 Poly(ADP-ribose) polymerase-1 (PARP-1) slides to accumulate at ends and prevent EXO1 binding. a Illustration of the DNA curtain assay with fluorescent PARP-1 (blue ovals). b Fluorescent PARP-1 (magenta) binding to DNA curtains in the presence (top) or absence (bottom) of buffer flow. Black circles indicate the diffusion barrier and arrows indicate the DNA ends. After PARP-1 was loaded, the DNA was stained with the fluorescent intercalating dye YoYo-1. c Histogram of the positions of 681 individual PARP-1 molecules along the length of the DNA substrate. Error bars were calculated by bootstrapping the data ${ }^{74}$ and indicate a $70 \%$ confidence interval. d Illustration (top) and kymograph (bottom) of two differently labeled PARP-1 (P1) molecules binding to the DNA end. PARP-1 reached the end by buffer flow-assisted one-dimensional sliding $88 \%$ of the time $(N=109 / 124)$.

e Representative kymographs of EXO1 resection of DNA pre-bound with PARP-1 (left) or PARP-2 (right). f Quantification of EXO1 loading events per DNA end bound by PARP-1 (left; $2.4 \pm 3.4 \% ; N=42$ PARP-1 molecules) or PARP-2 (right; $91.8 \pm 4.1 \% ; N=65$ PARP-2 molecules). Error bars represent mean $\pm \mathrm{s}$. e.m. ${ }^{\star \star \star \star} p \leq 0.0001$, (Student's $t$ test). $\mathbf{g}$ Model for PARP-1 inhibition of EXO1. P2 refers to PARP-2. YoYo-1 was omitted from all experiments described in c-f. h Recruitment of GFP-EXO1 at laser-induced double-strand breaks in HEK293T wild-type or PARP-1-/- cells. Source data are provided as a source data file 
a

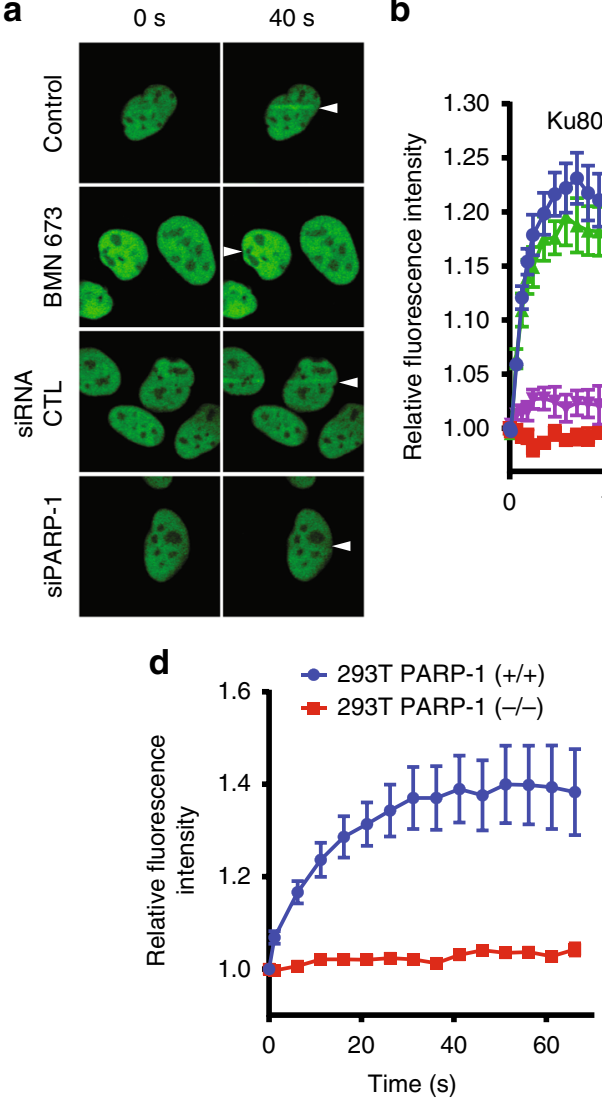

C

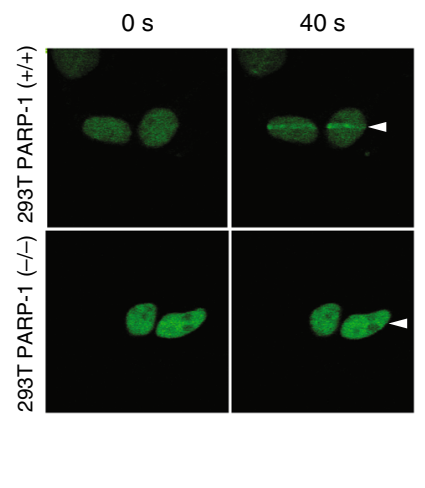

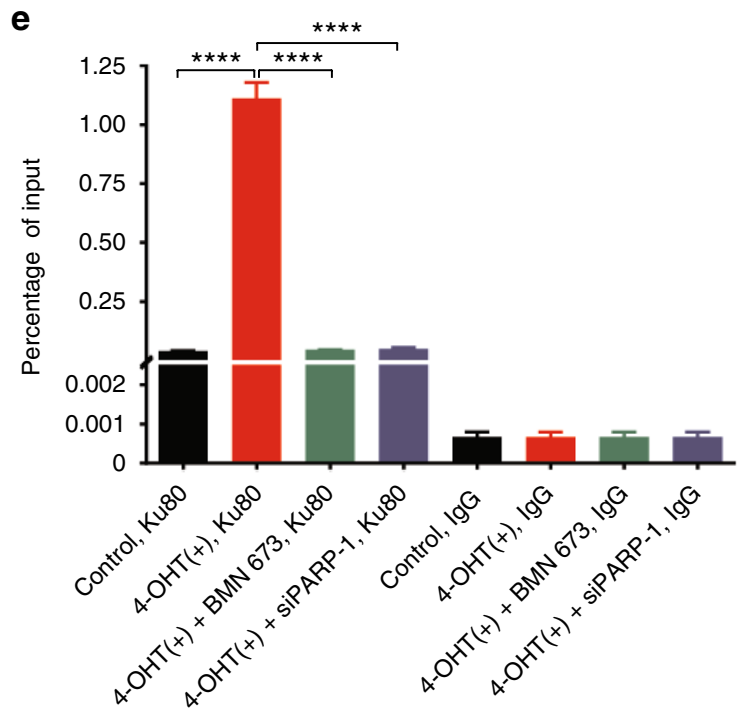

Fig. 6 Poly(ADP-ribose) polymerase-1 (PARP-1) knockdown or pharmacological inhibitor affects Ku80 recruitment on double-strand breaks. a U2OS cells stably expressing the Ku80-GFP were mock treated, treated with BMN 673, or silenced with PARP-1 siRNA and subjected to laser microirradiation-track studies. b Quantification of the relative fluorescence intensity of Ku80-GFP over time. Error bars represent mean with s.e.m. c Recruitment of Ku80-GFP in PARP-1-deficient CRISPR 293T cells is severely affected. d Quantification of the relative fluorescence intensity of Ku80-GFP over time. Error bars represent mean with s.e.m. e AsiS1-ER-U2OS reporter cells were cultured and treated with 4-hydroxytamoxifen ( $300 \mathrm{nM}$ ) for $1 \mathrm{~h}$. Soluble chromatin fractions were prepared and subjected to immunoprecipitation against IgG control and Ku80-specific antibody. Immunoprecipitated chromatins fractions were analyzed by quantitative polymerase chain reaction using specific primer pairs: chr1_89231183. Error bars represent mean \pm s.e.m. ${ }^{\star \star \star \star} p \leq 0.0001$ (Mann-Whitney $U$ test). Source data are provided as a source data file

RNA. We find that $\mathrm{Ku}$ association with the DSB is dependent on both PARP-1 and PARP-1 activity. Consequently, in addition to acting as a direct inhibitor of DNA end resection, PARP-1 also inhibits end resection by promoting $\mathrm{Ku}$ loading onto the DSB. To assess this further on other NHEJ components, we monitored phospho-DNA-PKcs foci formation in PARP-1 U2OS CRISPR/ Cas9-mediated knockout cells. Consistent with our previous studies showing a decrease of NHEJ in cellulo following ABT-888 treatment ${ }^{44}$, a decrease of phosphorylated DNA-PKcs foci was observed (Supplementary Fig. 7B). A Ku80 knockdown in HEK 293T cells leads to enhanced EXO1 recruitment (Supplementary Fig. 7C), suggesting that the recruitment of Ku80 by PARP-1 is a critical event for regulating EXO1-mediated DNA resection.

Increased resection tracks and HR in PARP-1-deficient cells. Next, we measured DNA resection in cellulo using a highresolution technique to see whether PARP-1 inhibition would lead to increased resection in different genetic contexts. The single-molecule analysis of resection tracks (SMART) assays is a very sensitive technique that can detect DNA resection upon IR, while almost no fibers can be detected without DNA damage ${ }^{28}$. It has been reported that BRCA1 is important to control the speed of DNA resection ${ }^{28}$ and that inhibition of $53 \mathrm{BP} 1$ in BRCA1-deficient cells rescue these cells through enhanced DNA resection ${ }^{45}$. Hence, our data predict that the DSB-induced overresection phenotype would not appear in BRCA1-deficient cells when challenged with the PARP-1 inhibitor BMN 673. However, the DNA resection machinery is intact in BRCA2-deficient cells, because BRCA2 acts later in HR, and we should observe this overresection phenotype under the same conditions. First, SMART assays revealed that PARP-1-deficient cells have longer resection tracks after IR treatment (Fig. 7a) and are IR sensitive in survival assays (Fig. 7b). When cells were treated with BMN 673 and IR, BRCA1 knockdown led to a decrease of BrdU accumulation, which could be partially rescued by 53BP1 knockdown (Supplementary Fig. 8A, B, D). SMART assays also recapitulated these results (Supplementary Figs. 8C, D and 9A). Conversely, BRCA2 


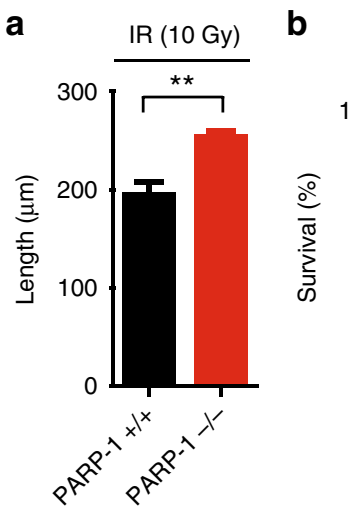

d

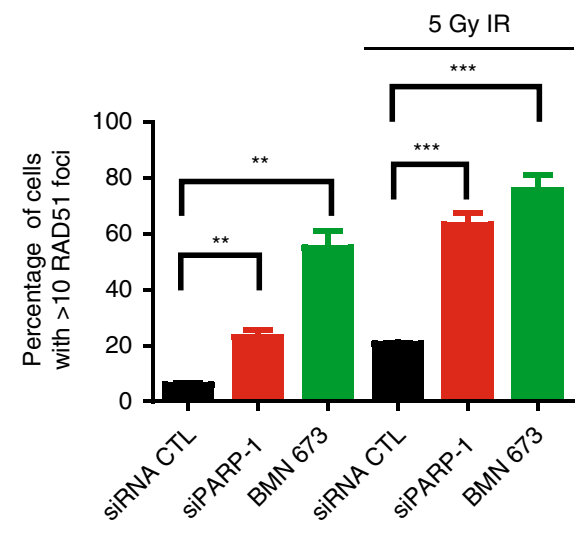

C

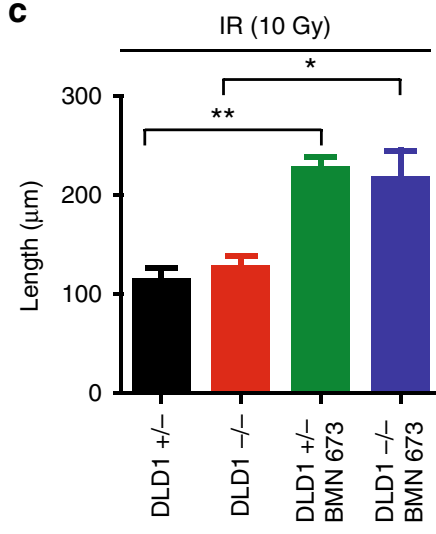

e

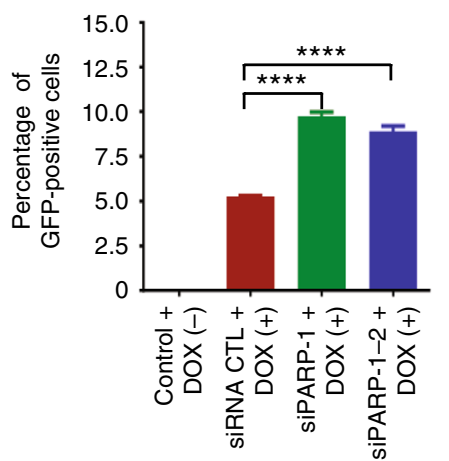

Fig. 7 Single-molecule analysis of resection tracks (SMART) analysis and effect of poly(ADP-ribose) polymerase-1 (PARP-1) on homologous recombination. a SMART of PARP-1-deficient mouse embryonic fibroblasts (MEFs). MEFs were treated with 10 Gy irradiation (IR). Error bars represent mean \pm s.e.m. b Treatment of HeLa cells with BMN 673 results in IR sensitivity. Error bars represent mean with s.e.m. c SMART of BRCA2-proficient (DLD1 BRCA2 (+/ $)$ ) and BRCA2-deficient cells (DLD1 BRCA2 $(-/-)$ ) either mock treated of treated with BMN $673(5 \mu \mathrm{M})$ were irradiated (10 Gy). Error bars represent Mean with s.e.m. d Attenuation of PARP-1 increases RAD51 foci in cellulo. Error bars represent mean \pm s.e.m. e Homologous recombination is increased in PARP-1 knockdown cells. Error bar represents mean \pm s.d. ${ }^{\star} p \leq 0.05,{ }^{\star \star} p \leq 0.01,{ }^{\star \star \star} p \leq 0.001,{ }^{\star \star \star \star} p \leq 0.0001$ (Mann-Whitney $U$ test). Source data are provided as a source data file

knockdown or DLD1 BRCA2 (-/-) cells showed a similar increase of BrdU staining (Supplementary Fig. 8A, B) or DSB resection tracks following treatment with BMN 673 (Fig. 7c) compared to the controls. These results show that PARP-1 inhibition-mediated over-resection of DSBs is achieved when cells have an effective DNA resection machinery.

Based on these results, we hypothesize that PARP-1 plays an important regulatory role in the DNA damage response by influencing DNA resection and consequently DNA repair. HR depends heavily on the extent of DNA resection. To address whether PARP-1 influences HR, we monitored RAD51 foci formation following $\gamma$-irradiation (Fig. 7d). In agreement with previous findings with an older generation of PARP-1 inhibitors $^{46}$, we observed that the percentage of cells harboring $>10$ RAD51 foci was increased in HEK 293T cells subjected to CRISPR/Cas9-mediated knockout of PARP-1 (Supplementary Fig. 9B, C) or in cells treated with a siRNA targeting PARP-1 or exposed to PARP-1 inhibition (Fig. 7d). In addition, using a doxycycline (Dox)-inducible I-SceI/DRGFP cell line, HR was increased with PARP-1 knockdown (Fig. 7e), BMN 673, or ABT 888-treated cells (Supplementary Fig. 9D). Collectively, these results show that PARP-1 negatively regulates HR in cellulo.

\section{Discussion}

In this study, we show evidence that PARP-1 antagonizes the activity of the MRN-RPA-BLM-EXO1 and MRN-RPA-BLM-DNA2 machineries for DNA DSB repair. Interestingly, PARP-1 mediates this effect through DNA end binding and promoting Ku80 loading. Furthermore, loss of PARP-1 leads to a decrease in the accumulation of HR suppressors 53BP1 and RIF1 at DSBs, which in turn increases the DNA accessibility to EXO1 resulting in excessive degradation of DNA lesions. Such an effect can be obtained with either PARP-1 knockdown or pharmacological inhibition of PARP1 activity. Thus mammalian cells have evolved several distinct regulatory systems that limit ssDNA overhang formation. First, a PARP-1-dependent mechanism influencing the Ku heterodimer and the 53BP1 pathway, and second, HELB that limits end resection in an RPA-dependent manner ${ }^{30}$. Recent studies have shown that DYNLL1 $^{47}$ and the Shieldin complex ${ }^{48-50}$ can also counteract DNA resection.

PARP-1 is an abundant nuclear chromatin-associated protein, well characterized for its high DNA damage-sensing ability. Once encountering free DNA ends, PARP-1 is catalytically activated and generates large amounts of PAR, which can function as a scaffold for the recruitment of a variety of DNA repair proteins ${ }^{12}$. It has been proposed that the local accumulation of PAR at DNA damage sites promotes liquid demixing, a phase separation event leading to compartmentalization of repair foci ${ }^{12}$. PAR polymers not only provide a loading platform for DDR-associated proteins and repair factors but also reprograms their functions through spatial and temporal interactions with their PAR reading motifs ${ }^{12,44}$. We envision that PARP-1 activation orchestrates the initial steps of DNA resection, granting access to the resection machineries. PARP-1 interacts 
with DNA-PKcs/Ku70/Ku $80^{51}$ and mediates this effect through DNA end binding and recruitment of the Ku complex to DNA ends. Although Ku and PARP-1 have been found to compete for binding to DNA end in vitro ${ }^{52}$, temporally, PARP-1 precedes Ku loading and its activity is required to load $\mathrm{Ku}$ onto DSB ends. This correlates well with the timing of PARP-1-mediated displacement of histones ${ }^{9}$, suggesting that PARP-1 activity is necessary to prepare chromatin for loading of $\mathrm{Ku}$ onto broken DSB ends. When PARP-1 is absent, neither PARP-1 nor Ku assemble to protect the DNA ends when analyzed by ChIP at nuclease-induced DSBs. Consequently, EXO1 has higher access to DSBs, and with a concomitant decrease of RIF1 and 53BP1, this leads to excessive DNA processing. In line with this observation, using the ER-AsiSI system, the group of Tanya Paull has shown that siKu86 or Ku86 conditional HCT116 cells show increased DNA resection ${ }^{42}$. This enhanced DNA resection has been observed in PARP-1 knockdown and PARP-1 knockout using SMART analysis, RPA staining as a surrogate marker of ssDNA accumulation, and native anti-BrdU staining. It is important to note that this excess of ssDNA is dependent on CtIP thereby confirming that the ssDNA detected is generated by DNA end resection. In a similar manner, we also observed excessive DSB processing in Veliparib- or BMN 673-treated cells. Initially, BMN 673 delays the displacement of PARP-1 on DSBs but does not prevent displacement, which reaches completion over a $10 \mathrm{~min}$ time frame following laser-induced DNA damage. At that point, it will no longer protect DSB ends, leading to a similar phenotype as a complete deletion of PARP-1 (Model in Fig. 8).
Using purified proteins, we also show that PARP-1 directly blocks both the EXO1 and DNA2 end resection machineries. Specifically, PARP-1 is able to slide to the end of DNA. This suggests a search mode employing 1D diffusion, which should stimulate the rate of recognition of newly formed DNA ends relative to three-dimensional diffusion mechanisms. Using singlemolecule microscopy, we determined that PARP-1, but not PARP-2, prevents the binding of EXO1 to DNA ends. The structural differences that allow PARP-1 but not PARP-2 or PARP-3 to inhibit end resection would be interesting to determine, given that PARP-1 physically occludes DNA ends from recognition by EXO1. The regulatory zinc finger domains that are unique to PARP-1 are likely to be key to this specificity of function. Our data suggest that, even prior to Ku loading, which strongly prevents the loading of EXO1, PARP-1 acts to block the end resection machinery. This observation fits into a model where both PARP-1 and Ku limit end resection, possibly by controlling the accumulation of the MRN complex ${ }^{53}$ and CtIP and that loss of either PARP-1 or Ku binding results in over-resection. We propose that this mechanism is conserved in pluricellular organisms, as the Iliakis group report that Chinese hamster ovary (CHO) Hamster cells treated with BMN 673 also have elevated DNA processing as measured by RPA foci ${ }^{54}$. PARP-2 has recently been shown to promote DNA resection ${ }^{55}$, but since BMN 673 inhibits PARP- ${ }^{56}$, it might not contribute to the over-resection phenotype observed in BMN673-inhibited cells.

Our work provides a conceptual framework to explain many observations reported in previous studies detailing the effects of PARP inhibition in a variety of contexts involving DNA
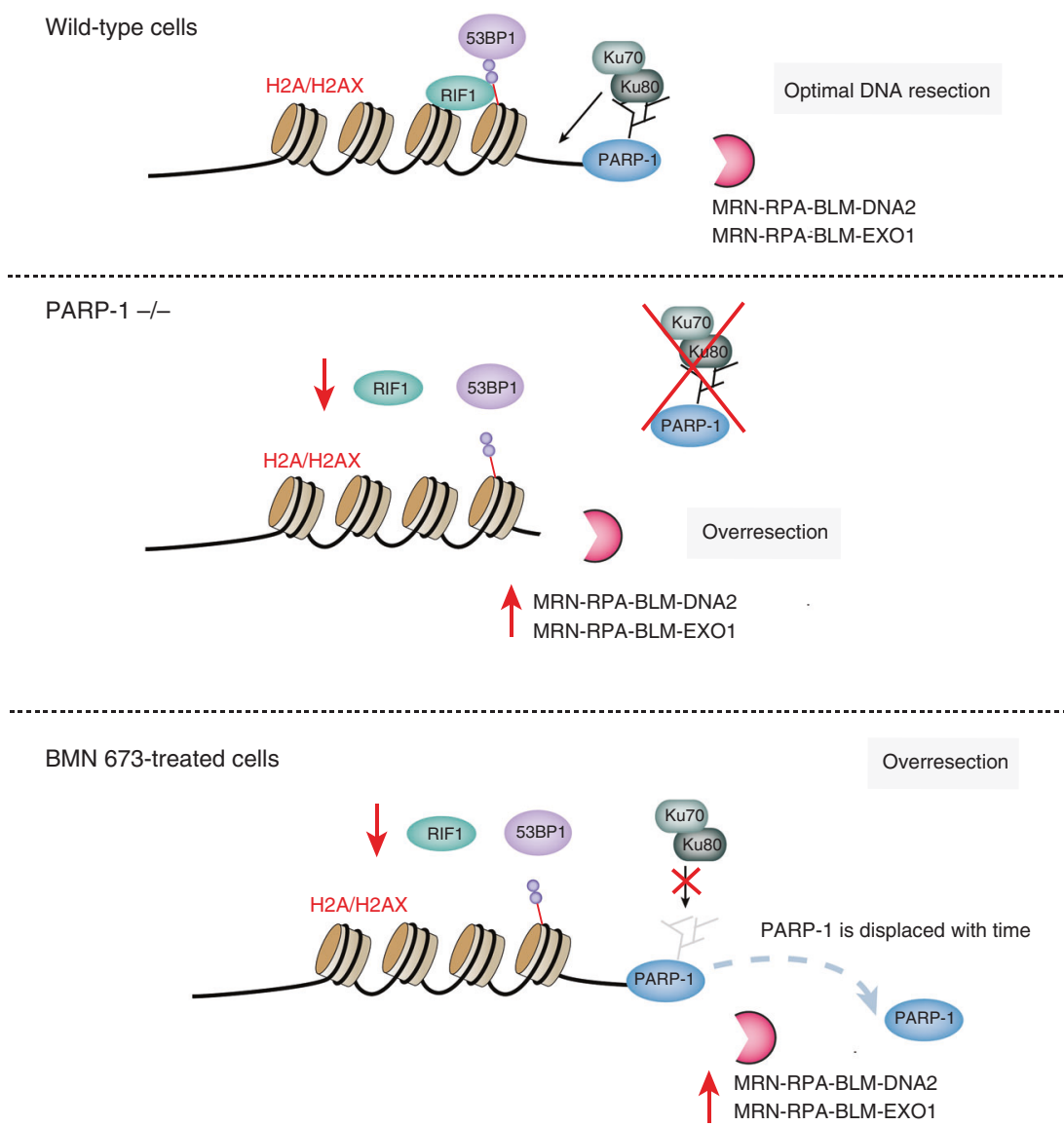

Fig. 8 Model. Following DNA damage, poly(ADP-ribose) polymerase-1 inhibition leads to a decrease of Ku80-end protection, RIF1 and 53BP1 foci formation, and increased DNA resection. Details are given in the text 
recombination transactions. Since the early 1980s, PARP-1 has been proposed to carry out an antirecombinogenic function. The group of Oikawa et al. first described a positive correlation between PARPis (benzamide and $m$-aminobenzamide) and induction of sister-chromatid exchanges (SCEs) in CHO-K1 cells $^{57}$. Hori demonstrated that reduced NAD as well as inhibition of PARP-1 (using 3-aminobenzamide) lead to a significant increase in SCEs in $\mathrm{CHO}$ cells ${ }^{58}$. Morrison et al. using PARP-1 ${ }^{-1}-$ mice, provided evidence of PARP-1 functions in maintaining genomic stability by demonstrating that PARP-1 is an anti-recombinogenic factor that inhibits ligation between the DNA termini exposed during $(\mathrm{V}(\mathrm{D}) \mathrm{J})$ recombination ${ }^{59}$. More recently, it was shown that PARP-1 PARylates BRCA1, and short- and long-track gene conversions, as well as chromosome aberrations after DNA damage, were increased by a BRCA1 PARylation mutant. In addition, treatment with olaparib also led to an enhancement of both types of HR frequencies ${ }^{60}$. Altogether, our data suggest that loss of PARP-1 facilitates HR, through enhanced DNA resection accounting for the (i) increase of sister chromatid exchanges ${ }^{57}$; (ii) anti-recombinogenic function of PARP- ${ }^{59}$; and (iii) increased HR repair by a BRCA1 PARylation mutant ${ }^{60}$. PARP-1 inhibition-induced HR is in accordance with our previous findings with another PARPi, ABT-888, which remained unexplained at the time $\mathrm{e}^{44}$. It also mimics the effect of a HELB knockdown, another DNA resection inhibitor ${ }^{30}$.

One of the first models proposed to explain the antitumor effects of PARPis in HR-deficient cells was based on the functions of PARP-1 in BER. This model postulated that catalytic inhibition of PARP-1 results in the accumulation of single-strand breaks that could not be repaired in HR-deficient cells. Two observations challenged this model. First, it was not possible to demonstrate increased single-strand breaks after PARP inhibition ${ }^{61}$, and synthetic lethality was not achieved when XRCC1, a key BER protein, was downregulated in BRCA2-deficient cells ${ }^{62}$. Hence, these observations raised the possibility that the effects of PARPis may be mediated through a mechanism distinct from BER. Consistent with this, Patel et al. have shown that deregulated NHEJ plays a major role in generating the genomic instability and cytotoxicity in HR-deficient cells treated with PARPis ${ }^{62}$.

We suggest that the observed synthetic lethality and cytotoxicity in different genetic contexts can be related to aberrant DNA resection as consequence of PARP-1 inhibition and DNA damage. Under these conditions, this phenotype will only be attained if DSBs are created and the DNA resection machinery is functional. This conclusion is highlighted by SMART analysis or BrdU staining of BRCA1- and BRCA2-deficient cells. Recent phase III studies have shown that PARPi activity extends beyond BRCA-related cancers for ovarian cancers devoid of known BRCA mutations, especially when platinum sensitivity and high-grade serous histology are present ${ }^{63}$. We propose that this effect could be due to misregulation of DNA resection. There are several ongoing clinical trials combining PARP-1 inhibitors with radiation therapy for which our study provides mechanistic insights into the tumor-killing activity observed in the clinic. Collectively, our results highlight that the functionality of DNA resection enzymes in response to DNA damage may be an important criterion to consider for the cell's ability to survive BMN 673 in the presence of DNA damage during clinical interventions in breast/ ovarian cancer and other solid tumors.

\section{Methods}

Cell lines, cell culture, drugs, and DNA constructs. Mouse embryonic fibroblasts proficient for PARP-1 (WT), or deficient for PARP-1 (PARP-1 (-/-)) were cultured in Dulbecco's modified Eagle's medium (DMEM) supplemented with 10\% fetal bovine serum (FBS) (Hyclone-ThermoFisher Scientific, Ottawa, Canada). U2OS, HeLa, and HeLa PARP-1 SilenciX control (Tebu-bio) were cultured in
DMEM with $10 \%$ FBS. PARP-1 HeLa SilenciX is a cell line engineered to stably knock down PARP-1 via RNA interference. Cells were maintained under hygromycin B selection $(250 \mu \mathrm{g} / \mathrm{mL}$; Invitrogen). U2OS-PARP-1 (-/-) cells were cultured in DMEM supplemented with $10 \%$ FBS and $2 \mu \mathrm{g} / \mathrm{mL}$ puromycin. U2OS cells stably expressing GFP-RPA2 were maintained in DMEM through continuous G418 selection $(500 \mu \mathrm{g} / \mathrm{mL}$; Invitrogen). The ER-AsiSI U2OS cell line was maintained in phenol red-free DMEM media supplemented with $10 \%$ charcoal-stripped FBS (Sigma) and $1 \mu \mathrm{g} / \mathrm{mL}$ puromycin. In order to induce DNA damage, AsiS1 U2OS cells were treated with $300 \mathrm{nM} 4$-OHT for $3 \mathrm{~h}$. U2OS cells stably expressing an mCherry-LacI-FokI construct containing an integrated reporter transgene were maintained in DMEM by puromycin $(2 \mu \mathrm{g} / \mathrm{mL})$ and hygromycin B $(100 \mu \mathrm{g} / \mathrm{mL})$ selection. To induce DNA DSBs, cell lines were treated with both $0.5 \mathrm{mM}$ Shield-1 and $1 \mathrm{mM}$ 4-OHT for $1 \mathrm{~h}$. Human embryonic kidney 293 cells (HEK 293), HEK 293T, or HEK 293T-PARP-1 (-/-) cells were cultured in DMEM supplemented with $10 \%$ FBS.

Generation of PARP-1 CRISPR/Cas9. U2OS cells were transfected with the appropriate guide RNA against PARP-1, cloned in a pSpCas9(BB)-2A-Puro (PX459) V2.0. The sgDNA sequence (5'-CGATGCCTATTACTGCACTG - $3^{\prime}$ ) was cloned at the BbsI site into pSpCas9(BB)-2A-Puro (PX459) V2.0 (Addgene plasmid ID: 62988). The positive clone was confirmed by restriction enzyme digestion (BbsI and $A g e \mathrm{I}$ ) of purified plasmids to check for insertion and further confirmed by Sanger sequencing. Twenty-four hours later, the transfected cells were selected in medium containing $2 \mu \mathrm{g} / \mathrm{mL}$ puromycin and then subcloned into 96 -well plates. Once at sufficient cell density, the subclones were analyzed for the presence of the target protein by western blotting (PARP-1).

Cell fractionation. Cell fractionation was carried out as described in ref. ${ }^{64}$ with slight modifications. Briefly, $3 \times 10^{6}$ HEK293T or CRISPR PARP-1 (-/-) cells per condition were collected and resuspended in $200 \mu \mathrm{L}$ of buffer A (10 mM HEPES $\mathrm{pH} 8.0,10 \mathrm{mM} \mathrm{KCl}, 1.5 \mathrm{mM} \mathrm{MgCl}_{2}, 0.34 \mathrm{M}$ sucrose, $10 \%$ glycerol, $1 \mathrm{mM}$ dithiothreitol (DTT), $1 \mathrm{mM}$ phenylmethanesulfonylfluoride (PMSF), $0.1 \%$ Triton$\mathrm{X}-100,10 \mathrm{mM} \mathrm{NaF}, 1 \mathrm{mM} \mathrm{Na} \mathrm{VO}_{4}$, supplemented with protease inhibitors) and kept for $5 \mathrm{~min}$ on ice. The soluble cytoplasmic fraction (S1) was separated from the nuclei (P2) by centrifugation for $4 \mathrm{~min}$ at $1300 \times g$ at $4{ }^{\circ} \mathrm{C}$. The nuclear fraction P2 was washed twice with $300 \mu \mathrm{L}$ buffer A, then resuspended in $200 \mu \mathrm{L}$ buffer B (3 mM EDTA, $0.2 \mathrm{mM}$ EGTA, $1 \mathrm{mM}$ DTT, $1 \mathrm{mM}$ PMSF, $10 \mathrm{mM} \mathrm{NaF}, 1 \mathrm{mM}$ $\mathrm{Na}_{3} \mathrm{VO}_{4}$, and protease inhibitors) and kept for $30 \mathrm{~min}$ on ice. The insoluble chromatin fraction (P3) was separated from nuclear soluble proteins (S3) by centrifugation for $4 \mathrm{~min}$ at $1700 \times g$ at $4{ }^{\circ} \mathrm{C}$ and washed three times with solution $\mathrm{B}$. $\mathrm{S} 1$ was cleared of insoluble proteins by centrifugation at $14,000 \times g$ for $15 \mathrm{~min}$ at $4{ }^{\circ} \mathrm{C}$ and the supernatant (S2) was kept for analysis. Cell fractions were subsequently analyzed by western blotting.

Antibodies, reagents, resources, and siRNAs. The antibodies used in this study as well as the working dilutions are listed in Supplementary Table 1. Key reagents or resources are listed in Supplementary Table 2. siRNAs are listed in Supplementary Table 3 .

Western blot analysis. Total cell lysates were prepared by lysing cells in RIPA buffer $(25 \mathrm{mM}$ Tris- $\mathrm{HCl}, 125 \mathrm{mM} \mathrm{NaCl}, 1 \%$ Nonidet-P-40, 0.5\% sodium deoxycholate, $0.1 \%$ sodium dodecyl sulfate (SDS), and a complete protease inhibitor cocktail (Roche)). Equal amounts of total protein were separated by SDS-polyacrylamide gel electrophoresis and then transferred to polyvinylidene difluoride membrane (BioRad) and immunoblotted with antibodies (Supplementary Table 1). When secondary antibodies conjugated with infrared-specific dyes (either Alexa Fluor 680 or Alexa Fluor 750) were used, fluorescence was imaged on the Odyssey Infrared Imaging system (LiCor Biosciences).

Transfection and siRNA. Transient siRNA transfections were carried out with Lipofectamine RNAiMax (Invitrogen) or Oligofectamine (Thermo Fisher Scientific) and analyses were performed $48-72 \mathrm{~h}$ after siRNA transfection. The siRNAs used in this study can be found in Supplementary Table 3.

Immunofluorescence staining. The effect of PARP-1 knockdown on IR-induced DNA end-resection was analyzed by immunofluorescence staining against RPA2 and BrdU. For RPA2 immunodetection, cells were pre-extracted with RPA buffer (25 mM HEPES pH 7.9, $300 \mathrm{mM}$ sucrose, $50 \mathrm{mM} \mathrm{NaCl}, 1 \mathrm{mM}$ EDTA, $3 \mathrm{mM}$ $\mathrm{MgCl}_{2}$, and $0.5 \%$ Triton $\mathrm{X}-100$ ) for $5 \mathrm{~min}$ on ice before being fixed at the indicated incubation time points after IR. This method removes nucleoplasmic signal and helps in the detection of foci. Cells were washed two times with phosphate-buffered saline (PBS) followed by fixation with $4 \%$ paraformaldehyde (w/v) in PBS for $15 \mathrm{~min}$ at room temperature. After two washes with PBS, cells were permeabilized in $0.5 \%$ Triton X-100 in PBS for 5 min. Cells were co-stained with primary antibodies against $\gamma$-H2AX (Active motif) and RPA2 (Abcam) in PBS for $1 \mathrm{~h}$ at room temperature. After three washes with PBS, cells were stained with goat anti-rabbit Alexa Fluor 488 and anti-mouse Cy3 secondary antibody (Molecular Probes, 1:400) in PBS for $30 \mathrm{~min}$ at room temperature. 
CtIP and Geminin immunofluorescence was performed as reported previously ${ }^{65}$. For RIF1, 53BP1, and cyclin A immunofluorescence staining, cells were either untreated or treated with $50 \mu \mathrm{M}$ etoposide for $1 \mathrm{~h}$ and fixed with $4 \%$ paraformaldehyde in PBS for $15 \mathrm{~min}$. Then cells were washed with TBS and fixed with cold methanol $\left(-20^{\circ} \mathrm{C}\right)$ for $5 \mathrm{~min}$ and permeabilized with PBS containing $0.2 \%$ Triton X-100 for $5 \mathrm{~min}$ and washed three times $5 \mathrm{~min}$ with TBS. The cells were quenched with $0.1 \%$ sodium borohydride for $5 \mathrm{~min}$, washed once with TBS, blocked in PBS containing 10\% goat serum and $1 \%$ bovine serum albumin (BSA) for $1 \mathrm{~h}$, and incubated with the primary antibody diluted in PBS $1 \%$ BSA for $2 \mathrm{~h}$ at room temperature. Coverslips were washed three times for $10 \mathrm{~min}$ with TBS before 1-h incubation with the appropriate secondary antibody conjugated to a fluorophore. Cells were rinsed again three times for $10 \mathrm{~min}$ with TBS. Coverslips were mounted onto slides with ProLong Gold (Thermo Fisher Scientific) antifade mountant with 4',6-diamidino-2-phenylindole (DAPI) (Life technologies).

For RAD51 and phosphoDNA-PKcs(S2056) immunostaining, cells were either untreated or treated with $5 \mathrm{~Gy}$ IR, released for $1 \mathrm{~h}$, and fixed with $4 \%$ paraformaldehyde in PBS for 25 min. Next, cells were permeabilized with PBS containing $0.5 \%$ Triton X-100 (PBS-T) for $15 \mathrm{~min}$ and washed three times with PBS $1 \times$. The cells were blocked in PBS containing $10 \%$ FBS for $1 \mathrm{~h}$ and incubated with the primary antibody (RAD51: 1:1000 or phosphoDNA-PKcs(S2056): 1:500) diluted in the blocking buffer for $2 \mathrm{~h}$ at room temperature. Coverslips were washed three times with PBS before 1-h incubation with the appropriate secondary antibody (1:1000) conjugated to a fluorophore again in blocking buffer. Cells were rinsed twice with PBS $1 \times$, then incubated in (1:1000) PBS-DAPI solution for $5 \mathrm{~min}$, and then washed twice with PBS $1 \times$. Coverslips were mounted onto slides with ProLong Gold antifade mountant.

\section{Recruitment of RPA and EXO1 to laser-induced DNA damage sites. The} evaluation of the recruitment kinetics of RPA to DNA damage sites was performed essentially as described ${ }^{6}$ with the exception of the following modifications. After overnight transfections with Effectene reagent (Qiagen), unsynchronized HEK 293 cells expressing the indicated RPA subunit fused to green fluorescent protein (GFP) and mCherry-PCNA fusion protein were incubated with fresh medium containing $1 \mu \mathrm{g} / \mathrm{mL}$ of Hoechst 33342 for $15 \mathrm{~min}$ at $37^{\circ} \mathrm{C}$ and treated with $5 \mu \mathrm{M}$ of PARPi BMN 673 (20 mM stock solution prepared in dimethyl sulfoxide (DMSO), Selleckchem) $1 \mathrm{~h}$ prior to microirradiation and recruitment analysis. A $37^{\circ} \mathrm{C}$ preheated stage with $5 \% \mathrm{CO}_{2}$ perfusion was used for time-lapse analysis on a Zeiss LSM-510 META NLO laser-scanning confocal microscope ( $\times 40$ objective). Localized DNA damage was generated along a defined region across the nucleus of a single living cell by using a bi-photonic excitation of the Hoechst 33342 dye, generated with a near-infrared 750-nm titanium:sapphire laser line (Chameleon Ultra II, Coherent Inc.) The laser output was set to $1.5 \%$ with 5 iterations. For each cell, 30 images were collected with a 5 sec interval. A Multi-Time macro developed in-house for the AIM software v3.2 (Zeiss) was used for image acquisition. Background and photobleaching corrections were applied to each dataset as described $^{10}$. The average accumulation \pm s.e.m. of RPA was plotted using a minimum of ten recruitment kinetic profiles per each RPA construct from three independent experiments. Only S-phase-positive PCNA (proliferating cell nuclear antigen) cells were chosen for recruitment ${ }^{66}$. Recruitment of GFP-EXO1 to laserinduced DSBs was performed as reported previously ${ }^{67}$.

\section{Recruitment of Ku80 to laser-induced DNA damage sites. For Ku80 laser} microirradiation experiments, a 1- $\mu \mathrm{m}$ diameter band of damage was introduced across the width of the nucleus. Background was determined based on measuring the fluorescence intensity outside of the cells (i.e., in regions containing only the growth medium). Fluorescence loss due to photobleaching that takes place during acquisition is removed by normalizing the total nuclear fluorescence to remain constant throughout the experiment. The fluorescence intensity of the damaged region was then monitored over time after correcting for background and fluorescence loss. The distribution of recruited protein can deviate from the initial band for two reasons. First, the distribution of chromatin determines whether or not the full width and full diameter of the band are sites of DNA damage. Second, as we have previously published, there is a decondensation of the damaged chromatin that causes the band to expand in width beyond the boundary of the original damaged area. Consequently, measurements restricted to the $1-\mu \mathrm{m}$ wide band where the laser microirradiation took place will slightly underestimate the extent of recruitment and chromatin-bound proteins that are unaffected will commonly show a very slight decrease in fluorescence intensity within the region.

BrdU/ssDNA assays. Cells were pre-incubated in the presence of $10 \mu \mathrm{M} \mathrm{BrdU}$ (Sigma) for $16 \mathrm{~h}$ followed by a 3 -h incubation after IR at $10 \mathrm{~Gy}$. Cells were subjected to in situ fractionation on ice for $10 \mathrm{~min}$ using sequential extraction with two different buffers. Pre-extraction buffer 1 (10 mM PIPES, pH 7.0, $300 \mathrm{mM}$ sucrose, $100 \mathrm{mM} \mathrm{NaCl}, 3 \mathrm{mM} \mathrm{MgCl}$, and $0.5 \%$ Triton-X100) and followed by preextraction buffer $2(10 \mathrm{mM}$ Tris pH 7.5, $10 \mathrm{mM} \mathrm{NaCl}, 3 \mathrm{mM} \mathrm{MgCl} 2,1 \%$ Nonidet P40 , and $0.5 \%$ sodium deoxycholate). Cells were washed three time with PBS followed by fixation with $4 \%$ paraformaldehyde $(\mathrm{w} / \mathrm{v}$ ) for $15 \mathrm{~min}$ at room temperature. Cells were washed with PBS and permeabilized in $0.5 \%$ Triton X-100 in PBS for $5 \mathrm{~min}$. Cells were incubated overnight at $4{ }^{\circ} \mathrm{C}$ with anti-BrdU antibody under non-denaturing conditions. In these native conditions, the anti-BrdU antibody only has access to its epitope in ssDNA. Unbound primary antibody was removed by washing in PBS at room temperature followed by incubation with the antimouse Cy3 secondary antibody for $30 \mathrm{~min}$ at room temperature. Slides were then washed for four times in PBS before mounting with Vectashield mounting medium (Vector Laboratories) containing DAPI. BrdU foci were observed by using an upright fluorescence microscope (Zeiss AxioImager.Z1) with a Plan Neofluar 1.3 N. A. $\times 40$ oil immersion objective. Image analysis was carried out by the ImageJ software (version 1.51k). The integrated intensity of individual BrdU foci and RPA2 foci were quantified by using GDSC ImageJ Find Foci plugins ${ }^{6}$.

ChIP assays. The effect of PARP-1 knockdown and PARP inhibition on RPA2, 53BP1, and RIF1 recruitment to a sequence-defined DSB site was determined quantitatively by ChIP followed by quantitative polymerase chain reaction (qPCR). Cells were crosslinked with $1 \%(\mathrm{v} / \mathrm{v})$ formaldehyde for $10 \mathrm{~min}$ and then glycine was added to a final concentration of $125 \mathrm{mM}$ for $5 \mathrm{~min}$ to stop the crosslinking reaction. Cells were lysed in lysis buffer (25 mM HEPES pH 7.9, $300 \mathrm{mM}$ sucrose, $50 \mathrm{mM} \mathrm{NaCl}, 1 \mathrm{mM}$ EDTA, $3 \mathrm{mM} \mathrm{MgCl}_{2}$, and $0.5 \%$ Triton X-100) and nuclei were isolated. Nuclear fractions were resuspended in sonication buffer $(50 \mathrm{mM}$ HEPES pH 7.9, $140 \mathrm{mM} \mathrm{NaCl}, 1 \mathrm{mM}$ EDTA, 1\% Triton X-100, 0.1\% sodium deoxycholate, $1 \%$ SDS, $1 \times$ protease inhibitor cocktail, and $1 \times$ phosphatase inhibitor cocktail (Roche)) for $10 \mathrm{~min}$ on ice and sonicated to obtain approximately 200-500-bp chromatin fragments using a Bioruptor (Diagenode). Chromatin fragments were precleared with magnetic Dynabeads protein G (Life Technologies) for $1 \mathrm{~h}$ and incubated with pre-bound antibody-Dynabeads protein $\mathrm{G}$ overnight at $4{ }^{\circ} \mathrm{C}$. Beads were washed once in low-salt buffer $(20 \mathrm{mM}$ Tris, $\mathrm{pH}$ 8.1, $2 \mathrm{mM}$ EDTA, $50 \mathrm{mM}$ $\mathrm{NaCl}, 1 \%$ Triton X- 100 , and $0.1 \% \mathrm{SDS})$, once in high-salt buffer $(20 \mathrm{mM}$ Tris, $\mathrm{pH}$ $8.1,2 \mathrm{mM}$ EDTA, $500 \mathrm{mM} \mathrm{NaCl}, 1 \%$ Triton X-100, and $0.1 \%$ SDS), once in $\mathrm{LiCl}$ buffer (10 mM Tris, pH 8.0, $1 \mathrm{mM}$ EDTA, $0.25 \mathrm{mM} \mathrm{LiCl}, 1 \%$ Nonidet P-40, and 1\% deoxycholic acid), and twice in TE buffer $(10 \mathrm{mM}$ Tris- $\mathrm{HCl}, \mathrm{pH} 8.0$, and $1 \mathrm{mM}$ EDTA). Washed beads were eluted twice with $100 \mu \mathrm{L}$ of elution buffer (1\% SDS and $0.1 \mathrm{M} \mathrm{NaHCO}_{3}$ ) and crosslinks were reversed by overnight incubation at $65^{\circ} \mathrm{C}$ in $0.1 \mathrm{mg} / \mathrm{mL}$ RNase A, $0.3 \mathrm{M} \mathrm{NaCl}$, and $0.2 \mathrm{mg} / \mathrm{mL}$ proteinase $\mathrm{K}$. The DNA samples were purified with Qiaquick PCR columns (Qiagen). qPCR was carried out on an Applied Biosystem 7900 HT Fast instrument using the SYBR Green detection system. The results of the quantitative ChIP assays are the mean with s.e.m. of qPCR reactions from three independent experiments and primers used are listed in Supplementary Tables 4 and 5 .

ER-AsiSI resection assay. The percentage of resection adjacent to a specific DSB1 (Chr 1: 89231183) was measured as described ${ }^{42}$ with some modifications. The primer pairs for "DSB1" and "DSB2" are across BsrG1 and BamH1 restriction sites, respectively. Briefly, ER-AsiSI U2OS cells were treated with $300 \mathrm{nM}$ of 4-OHT (Sigma) for $3 \mathrm{~h}$ to allow the nuclear translocation of AsiSI and the induction of DSBs. Cells were collected and genomic DNA was extracted and digested with $B s r \mathrm{GI}$ or $\mathrm{BamH1}$ enzymes or mock digested overnight at $37^{\circ} \mathrm{C}$. Digested or mockdigested samples were used as a template for $\mathrm{qPCR}$ performed using SYBR Green master mix. Primers used are listed in Supplementary Table $6^{42}$. For each sample, a $\Delta \mathrm{Ct}$ was calculated by subtracting the $\mathrm{Ct}$ value of the mock-digested sample from the $\mathrm{Ct}$ value of the digested sample.

Protein purification. PARP-1, PARP-2, and PARP-3 were purified according to standard procedures ${ }^{69,70}$. BLM was tagged at the N-terminus with GST and at the C-terminus with $\mathrm{His}_{10}$ and purified as described for PALB2 ${ }^{70}$. MRE11-RAD50NBS1 was purified according to an established protocol ${ }^{71}$. RPA was purified as described ${ }^{72}$. Human EXO1 ${ }^{30}$ or biotinylated EXO1 for single-molecule experiments was purified as described ${ }^{73}$. For recombinant DNA2 protein purification, Sf9 insect cells $\left(1 \mathrm{~L}\right.$ at $10^{6}$ cells $\left./ \mathrm{mL}\right)$ were infected with a GST-DNA2-FLAG baculovirus. At $48 \mathrm{~h}$ post-infection, cells were harvested by centrifugation and the pellet was frozen on dry ice. Cells were lysed in Buffer 1 (1× PBS containing $150 \mathrm{mM}$ $\mathrm{NaCl}, 1 \mathrm{mM}$ EDTA, $0.05 \%$ Triton X-100, $1 \mathrm{mM}$ DTT, and protease inhibitors) and homogenized by 20 passes through a Dounce homogenizer (pestle A). The cell lysate was incubated with $1 \mathrm{mM} \mathrm{MgCl}$ and $2.5 \mathrm{U} / \mathrm{mL}$ benzonase nuclease at $4{ }^{\circ} \mathrm{C}$ for $1 \mathrm{~h}$ followed by centrifugation at $93,753 \times g$ for $1 \mathrm{~h}$. The soluble cell lysate was incubated with $1 \mathrm{~mL}$ of GST-Sepharose beads for $90 \mathrm{~min}$ at $4{ }^{\circ} \mathrm{C}$ with gentle rotation. The beads were washed twice with buffer 1 followed by incubation with buffer 2 (Buffer 1 with $5 \mathrm{mM} \mathrm{ATP,} 15 \mathrm{mM} \mathrm{MgCl}$ ) for $1 \mathrm{~h}$ at $4{ }^{\circ} \mathrm{C}$. Sepharose GST beads were washed twice with buffer 1 supplemented with $200 \mathrm{mM} \mathrm{NaCl}$ and once with P5 buffer $\left(50 \mathrm{mM} \mathrm{NaHPO}_{4} \mathrm{pH} 7.0,500 \mathrm{mM} \mathrm{NaCl}, 10 \%\right.$ glycerol, $0.05 \%$ Triton-X-100, $5 \mathrm{mM}$ imidazole) followed by cleavage with PreScission protease (60 U/mL, GE Healthcare Life Sciences), overnight at $4{ }^{\circ} \mathrm{C}$ in P5 buffer. The supernatant was then collected and completed to $10 \mathrm{~mL}$ with Flag-binding buffer (50 mM Tris- $\mathrm{HCl} \mathrm{pH} 7.5,150 \mathrm{mM} \mathrm{NaCl}, 1 \mathrm{mM}$ EDTA, $10 \%$ glycerol, $0.025 \%$ Triton X-100) before incubation with $600 \mu \mathrm{L}$ of M2 anti-Flag affinity gel (Invitrogen) for $1 \mathrm{~h}$ at $4{ }^{\circ} \mathrm{C}$. The beads were washed twice with washing buffer (Flagbinding buffer supplemented with $100 \mathrm{mM} \mathrm{NaCl}$ ). After two additional washes with Flag Elution buffer $(50 \mathrm{mM}$ Tris- $\mathrm{HCl} \mathrm{pH} 7.5,150 \mathrm{mM} \mathrm{NaCl}, 0.025 \%$ Triton $\mathrm{X}-100$, and $10 \%$ glycerol), proteins were eluted twice in one volume of beads with Flag Elution buffer and $500 \mu \mathrm{g} / \mathrm{mL}$ of $3 \times$-Flag peptide for $45 \mathrm{~min}$ at $4{ }^{\circ} \mathrm{C}$. Protein 
were then dialyzed in the storage buffer $(20 \mathrm{mM}$ Tris- $\mathrm{HCl}, \mathrm{pH} 7.4,200 \mathrm{mM} \mathrm{NaCl}$, $10 \%$ glycerol, $1 \mathrm{mM} \mathrm{DTT}$ ) and stored in aliquots at $-80^{\circ} \mathrm{C}$.

DNA resection assays. Assays were performed with pUC19 DNA linearized with $K p n \mathrm{I}$ and then $3^{\prime}$ labeled with $\left[{ }^{-}{ }^{32} \mathrm{P}\right]$ ATP and terminal deoxytransferase (NEB). For the DNA2 resection machinery, reactions were conducted using $50 \mathrm{nM}$ of substrate in standard buffer (20 mM Na-HEPES pH 7.5, $0.1 \mathrm{mM}$ DTT, $0.05 \%$ Triton X-100, $100 \mu \mathrm{g} / \mathrm{mL}$ BSA). Two millimolar ATP and $5 \mathrm{mM} \mathrm{MgCl}_{2}$ were added to the reaction buffer immediately before reconstitution of the resection machineries. The reactions were initiated on ice by adding either NAD, PARP-1, PARP-2 or PARP-3 as indicated in the figure and transferred immediately to $37^{\circ} \mathrm{C}$. After 5 $\mathrm{min}$, the order of addition and incubation of the respective protein components were: MRN (10 nM, $5 \mathrm{~min})$, RPA ( $100 \mathrm{nM}, 5 \mathrm{~min})$, BLM ( $15 \mathrm{nM}, 3 \mathrm{~min})$ and DNA2 $(15 \mathrm{nM}, 45 \mathrm{~min})$. For the EXO1 resection machinery, reactions were conducted using resection buffer $(25 \mathrm{mM}$ MOPS pH 7, $60 \mathrm{mM} \mathrm{KCl}, 1 \%$ Tween $20,2 \mathrm{mM}$ DTT, $5 \mathrm{mM} \mathrm{MgCl} 2,2 \mathrm{mM} \mathrm{ATP}$ ) and the same proteins and time of incubation as mentioned above except for EXO1 at a concentration of $10 \mathrm{nM}$ instead of DNA2. Reactions were followed by proteinase $\mathrm{K}$ treatment for $30 \mathrm{~min}$ at $37^{\circ} \mathrm{C}$. Products were analyzed on a $1 \%$ native agarose gel. Gels were dried on DE81 paper (Whatman) and signals were detected by autoradiography. Densitometric analyses were performed using the FLA-5100 phosphorimager (Fujifilm) and quantified using the Image Reader FLA-5000 v1.0 software.

DNA-binding assays. The DNA-binding reactions $(10 \mu \mathrm{L})$ contained ${ }^{32} \mathrm{P}$-labeled DNA oligonucleotides $(100 \mathrm{nM})$ and the indicated concentrations of PARP-1 and NAD in resection buffer $(20 \mathrm{mM}$ Na-HEPES pH 7.5, $0.1 \mathrm{mM}$ DTT, $0.05 \%$ Triton $\mathrm{X}-100,100 \mu \mathrm{g} / \mathrm{mL}$ BSA, $5 \mathrm{mM} \mathrm{MgCl} 2,2 \mathrm{mM}$ ATP). Reaction mixtures were incubated at $37^{\circ} \mathrm{C}$ for $10 \mathrm{~min}$ and then protein-DNA complexes were fixed with $0.2 \%(\mathrm{v} / \mathrm{v})$ glutaraldehyde for $15 \mathrm{~min}$. The reactions were subjected to electrophoresis on an $8 \% 1 \times$-TBE-acrylamide gel and ${ }^{32} \mathrm{P}$-labeled DNA was visualized by autoradiography. Sequences of the oligonucleotides are found in Supplementary Table 7 .

Single-molecule imaging and DNA curtains. Single-molecule DNA curtain data were collected at $37^{\circ} \mathrm{C}$ using a Nikon Ti-E microscope in a prism-TIRF configuration. Data were collected with a $200-\mathrm{ms}$ exposure through a $\times 60$ waterimmersion objective (1.2 NA, Nikon), a 500-nm long-pass (Chroma), and a 638$\mathrm{nm}$ dichroic beam splitter (Chroma) for two-channel detection through two EMCCD cameras (Andor iXon DU897, cooled to $-80^{\circ} \mathrm{C}$ ). Histograms of PARP-1binding preference on DNA were acquired by fitting a two-dimensional Gaussian to each individual molecule and finding the center of the fit relative to the barrier position using a custom-written FIJI script (available upon request). A Gaussian curve with a constant offset was fit to the largest peak of the histogram using a custom script written in MATLAB (available upon request). The constant offset in the fitting accounts for molecules that bind nonspecifically along the length of the DNA substrate. The center of the fit is reported in the corresponding figure panel.

For DNA curtains, microscope slides with microfabricated chromium barriers were encased in a microfluidic flowcell and passivated with a fluid lipid bilayer. DNA molecules $(\lambda, 48.5 \mathrm{~kb})$ with biotinylated ends were bound to a subset of the lipids via a biotin-streptavidin interaction. The flowcells were attached by nanoports to a syringe pump-controlled microfluidics system.

$\lambda$-DNA substrates for DNA curtains were purchased from NEB and annealed with two oligonucleotides: IF7: (5'-[p]AGG TCG CCG CCC[Biotin]- $3^{\prime}$ ) and LM3: (5'- [p]GGG CGG CGA CCT TTT TTT TTT TTT TTT TTT TTT TTT TTT TTT TTT TTT TTT TTT TTT TTT TTT TTT TTT TTT TTT TTT TTT TTT TTT TTT $\left.-3^{\prime}\right)$. This generated a substrate that had a biotin for attaching to the flowcell surface on one side and a 78-nt $3^{\prime}$ overhang on the other side, which was suitable for Exo1 loading. For visualizing the DNA, YoYo-1 was injected into the flowcell with a glucose oxidase/catalase mixture.

To perform PARP-1 labeling, $300 \mathrm{nM}$ mouse anti-6xHis antibody (Clontech) was pre-incubated with $400 \mathrm{nM}$ anti-mouse secondary QDots (Invitrogen, 605 or 705 depending on labeling strategy) in a $5 \mu \mathrm{L}$ volume for $10 \mathrm{~min}$ on ice. PARP-1 or PARP-2 were incubated with the antibody mixture for another 10 min on ice and diluted to a final volume of $200 \mu \mathrm{L}$ ( $6.25 \mathrm{nM}$ PARP, $7.5 \mathrm{nM}$ anti-His antibody, $10 \mathrm{nM}$ QDots; final concentrations). PARP-1 or PARP-2 were injected onto the microscope

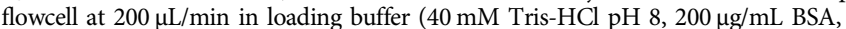
$2 \mathrm{mM}$ DTT, $2 \mathrm{mM} \mathrm{MgCl}_{2}$ ). After binding, the flowcell was switched to EXO1 resection buffer ( $40 \mathrm{nM}$ Tris- $\mathrm{HCl} \mathrm{pH} 8,60 \mathrm{mM} \mathrm{NaCl}, 200 \mu \mathrm{g} / \mathrm{mL}$ BSA, $2 \mathrm{mM}$ DTT, $2 \mathrm{mM} \mathrm{MgCl} 2,1 \mathrm{mM}$ ATP) for subsequent EXO1 loading.

EXO1 loading was performed by pre-incubating $100 \mathrm{nM}$ Streptavidin QDots 605 with $80 \mathrm{nM}$ EXO1-biotin in $10 \mu \mathrm{L}$ EXO1 loading buffer $(40 \mathrm{mM}$ Tris- $\mathrm{HCl} \mathrm{pH}$ $8,60 \mathrm{mM} \mathrm{NaCl}, 200 \mu \mathrm{g} / \mathrm{mL}$ BSA, $2 \mathrm{mM}$ DTT, $2 \mathrm{mM} \mathrm{MgCl} 2,1 \mathrm{mM} \mathrm{ATP}$ ) on ice for $10 \mathrm{~min}$. Then the mixture was diluted to a final volume of $200 \mu \mathrm{L}$ in EXO1 loading buffer plus free biotin (4 $\mathrm{nM}$ EXO1, $5 \mathrm{nM}$ QDots) and injected onto DNA curtains at $200 \mu \mathrm{L} / \mathrm{min}$. To prevent dye-induced changes in protein-DNA interactions, YoYo-1 was omitted in experiments involving PARP-1/EXO1 and PARP-2/EXO1.

Single-molecule analysis of resection tracks. The indicated cell lines or siRNA transfected cells were incubated with $10 \mu \mathrm{M}$ BrdU for $48 \mathrm{~h}$. After $48 \mathrm{~h}$, the cells were treated with $5 \mu \mathrm{M}$ BMN 673 for $1 \mathrm{~h}$ before IR ( $10 \mathrm{~Gy}$ and released for $1 \mathrm{~h}$ ). Cells were harvested and counted, $1 \times 10^{5}$ cells per condition were spun down, and resuspended in the resuspension buffer from the FiberPrep DNA Extraction Kit (Genomic Vision). Agarose plugs and DNA solutions were made according to the FiberPrep DNA extraction Kit. Using the FiberComb (Genomic Vision), the DNA was stretched onto a Combicoverslip. The cover slips were baked for $2 \mathrm{~h}$ at $60^{\circ} \mathrm{C}$, then incubated in primary antibody (1:100 anti-BrdU) in 5\% PBS-BSA for $2 \mathrm{~h}$ at $37^{\circ} \mathrm{C}$. The coverslips were washed with PBS-T 3 times for 3 min with shaking and incubated for $1 \mathrm{~h}$ in the appropriate secondary antibody $(1: 200)$ conjugated to a fluorophore in a humidified chamber at $37^{\circ} \mathrm{C}$. The slides were washed with PBS-T 3 times for $3 \mathrm{~min}$. If needed, $\mathrm{YOYO}^{\mathrm{m}}$-1 Iodide staining (1:1000) was performed for $10 \mathrm{~min}$. The slides were washed with $\mathrm{ddH}_{2} \mathrm{O}$ for $1 \mathrm{~min}$. The DNA is then dehydrated by submerging it for $1 \mathrm{~min}$ in $70 \%, 90 \%$, and $100 \%$ ethanol sequentially and visualized on a DMI6000B microscope. Fiber length was evaluated by Image J analysis (version $1.51 \mathrm{k}$ ).

Survival assays. Cells were seeded in triplicates into a Corning 3603 black-sided clear bottom 96-well microplate at a density of 2000 cells per well. Once attached, the media was changed to include the desired concentration of BMN 673. One hour after BMN 673 treatment, the plate was irradiated with 7.5 Gy. The plates were incubated for $120 \mathrm{~h}$. The nuclei were stained with Hoechst 33342 (Invitrogen) at $10 \mu \mathrm{g} / \mathrm{mL}$ in media for $30 \mathrm{~min}$ at $37^{\circ} \mathrm{C}$. Images of entire wells were acquired at $\times 4$ with a Cytation 5 Cell Imaging Multi-Mode Reader followed by quantification of Hoechst-stained nuclei with the Gen5 Data Analysis Software v3.03 (BioTek Instruments). Cell viability was expressed as the percentage of survival in BMN673treated cells relative to vehicle (DMSO)-treated cells.

HR in cellulo reporter assays. Dox-inducible I-SceI/DRGFP cell line (TRI-DRU2OS) was treated without/with $10 \mu \mathrm{g} / \mathrm{mL}$ Dox and BMN $673(1 \mu \mathrm{M})$ or ABT-888 $(5 \mu \mathrm{M})$ and incubated for $48 \mathrm{~h}$. HR efficiencies were analyzed by flow cytometry after 48-h incubation. HR efficiency was expressed as the percentage of GFP-positive cells. Samples were analyzed in triplicate. Values are expressed as mean and s.e.m.

Statistical analyses. All data are representative of three or more independent experiments. Prism ver 6.0 was used to do the statistical analyses.

Reporting summary. Further information on research design is available in the Nature Research Reporting Summary linked to this article.

\section{Data availability}

The source data and uncropped gel pictures underlying Figs. 1A-G, 2B-D, 3A, B, D, E, 4A-F, 5C, F, H, 6B, D, E, and 7A-E and Supplementary Figs. 1A, B, 2A, B, 3A-G, 4A, B D, E, 5A-F, 6A-G, 7A-C, 8B-D, and 9B-D are provided as a source data file. Data of this study are available from the authors upon reasonable request.

\section{Received: 23 September 2018 Accepted: 29 May 2019}

Published online: 04 July 2019

\section{References}

1. Vilenchik, M. M. \& Knudson, A. G. Endogenous DNA double-strand breaks: production, fidelity of repair, and induction of cancer. Proc. Natl Acad. Sci. USA 100, 12871-12876 (2003).

2. Ciccia, A. \& Elledge, S. J. The DNA damage response: making it safe to play with knives. Mol. Cell 40, 179-204 (2010).

3. Jungmichel, S. et al. Proteome-wide identification of poly(ADP-Ribosyl)ation targets in different genotoxic stress responses. Mol. Cell 52, 272-285 (2013).

4. Zhang, Y., Wang, J., Ding, M. \& Yu, Y. Site-specific characterization of the Aspand Glu-ADP-ribosylated proteome. Nat. Methods 10, 981-984 (2013).

5. Gagne, J. P. et al. Quantitative site-specific ADP-ribosylation profiling of DNA-dependent PARPs. DNA Repair (Amst.) 30, 68-79 (2015).

6. Gagne, J. P. et al. Quantitative proteomics profiling of the poly(ADP-ribose)related response to genotoxic stress. Nucleic Acids Res. 40, 7788-7805 (2012).

7. Gibson, B. A. et al. Chemical genetic discovery of PARP targets reveals a role for PARP-1 in transcription elongation. Science 353, 45-50 (2016).

8. Wacker, D. A., Frizzell, K. M., Zhang, T. \& Kraus, W. L. Regulation of chromatin structure and chromatin-dependent transcription by poly(ADPribose) polymerase-1: possible targets for drug-based therapies. Subcell. Biochem. 41, 45-69 (2007).

9. Strickfaden, H. et al. Poly(ADP-ribosyl)ation-dependent transient chromatin decondensation and histone displacement following laser microirradiation. J. Biol. Chem. 291, 1789-1802 (2016).

10. Haince, J. F. et al. PARP1-dependent kinetics of recruitment of MRE11 and NBS1 proteins to multiple DNA damage sites. J. Biol. Chem. 283, 1197-1208 (2008).

11. Krietsch, J. et al. Reprogramming cellular events by poly(ADP-ribose)-binding proteins. Mol. Aspects Med. 34, 1066-1087 (2013). 
12. Altmeyer, M. et al. Liquid demixing of intrinsically disordered proteins is seeded by poly(ADP-ribose). Nat. Commun. 6, 8088 (2015).

13. D’Amours, D., Sallmann, F. R., Dixit, V. M. \& Poirier, G. G. Gain-of-function of poly(ADP-ribose) polymerase-1 upon cleavage by apoptotic proteases: implications for apoptosis. J. Cell Sci. 114, 3771-3778 (2001).

14. Slade, D. et al. The structure and catalytic mechanism of a poly(ADP-ribose) glycohydrolase. Nature 477, 616-620 (2011).

15. Maya-Mendoza, A. et al. High speed of fork progression induces DNA replication stress and genomic instability. Nature 559, 279-284 (2018).

16. Hanzlikova, H. et al. The importance of poly(ADP-ribose) polymerase as a sensor of unligated Okazaki fragments during DNA replication. Mol. Cell 71, 319-331 e313 (2018)

17. Zimmermann, M. et al. CRISPR screens identify genomic ribonucleotides as a source of PARP-trapping lesions. Nature 559, 285-289 (2018).

18. Ame, J. C., Spenlehauer, C. \& de Murcia, G. The PARP superfamily. Bioessays 26, 882-893 (2004).

19. Ray Chaudhuri, A. \& Nussenzweig, A. The multifaceted roles of PARP1 in DNA repair and chromatin remodelling. Nat. Rev. Mol. Cell Biol. 18, 610-621 (2017).

20. Rulten, S. L. et al. PARP-3 and APLF function together to accelerate nonhomologous end-joining. Mol. Cell 41, 33-45 (2011).

21. De Murcia, J. M. et al. Requirement of poly(ADP-ribose) polymerase in recovery from DNA damage in mice and in cells. Proc. Natl Acad. Sci. USA 94, 7303-7307 (1997).

22. Couto, C. A.-M. et al. PARP regulates nonhomologous end joining through retention of $\mathrm{Ku}$ at double-strand breaks. J. Cell Biol. 194, 367-375 (2011)

23. Langelier, M. F., Planck, J. L., Roy, S. \& Pascal, J. M. Crystal structures of poly (ADP-ribose) polymerase-1 (PARP-1) zinc fingers bound to DNA: structural and functional insights into DNA-dependent PARP-1 activity. J. Biol. Chem. 286, 10690-10701 (2011).

24. Langelier, M. F., Ruhl, D. D., Planck, J. L., Kraus, W. L. \& Pascal, J. M. The $\mathrm{Zn} 3$ domain of human poly(ADP-ribose) polymerase-1 (PARP-1) functions in both DNA-dependent poly(ADP-ribose) synthesis activity and chromatin compaction. J. Biol. Chem. 285, 18877-18887 (2010).

25. Langelier, M. F., Zandarashvili, L., Aguiar, P. M., Black, B. E. \& Pascal, J. M. $\mathrm{NAD}(+)$ analog reveals PARP-1 substrate-blocking mechanism and allosteric communication from catalytic center to DNA-binding domains. Nat. Commun. 9, 844 (2018).

26. Yun, M. H. \& Hiom, K. CtIP-BRCA1 modulates the choice of DNA doublestrand-break repair pathway throughout the cell cycle. Nature 459, 460-463 (2009).

27. Nakamura, K. et al. Collaborative action of Brcal and CtIP in elimination of covalent modifications from double-strand breaks to facilitate subsequent break repair. PLoS Genet. 6, e1000828 (2010).

28. Cruz-Garcia, A., Lopez-Saavedra, A. \& Huertas, P. BRCA1 accelerates CtIPmediated DNA-end resection. Cell Rep. 9, 451-459 (2014).

29. Nimonkar, A. V. et al. BLM-DNA2-RPA-MRN and EXO1-BLM-RPA-MRN constitute two DNA end resection machineries for human DNA break repair. Genes Dev. 25, 350-362 (2011).

30. Tkac, J. et al. HELB is a feedback inhibitor of DNA end resection. Mol. Cell 61, 405-418 (2016).

31. Zimmermann, M., Lottersberger, F., Buonomo, S. B., Sfeir, A. \& de Lange, T. 53BP1 regulates DSB repair using Rif1 to control 5' end resection. Science 339, 700-704 (2013).

32. Escribano-Diaz, C. et al. A cell cycle-dependent regulatory circuit composed of 53BP1-RIF1 and BRCA1-CtIP controls DNA repair pathway choice. Mol. Cell 49, 872-883 (2013).

33. Isabelle, M. et al. Investigation of PARP-1, PARP-2, and PARG interactomes by affinity-purification mass spectrometry. Proteome Sci. 8, 22 (2010).

34. LaFargue, C. J., Dal Molin, G. Z., Sood, A. K. \& Coleman, R. L. Exploring and comparing adverse events between PARP inhibitors. Lancet Oncol. 20, e15-e28 (2019)

35. Drean, A., Lord, C. J. \& Ashworth, A. PARP inhibitor combination therapy. Crit. Rev. Oncol. Hematol. 108, 73-85 (2016).

36. Mortusewicz, O., Ame, J. C., Schreiber, V. \& Leonhardt, H. Feedbackregulated poly(ADP-ribosyl)ation by PARP-1 is required for rapid response to DNA damage in living cells. Nucleic Acids Res. 35, 7665-7675 (2007).

37. Aleksandrov, R. et al. Protein dynamics in complex DNA lesions. Mol. Cell 69, 1046-1061 e1045 (2018).

38. Murai, J. et al. Trapping of PARP1 and PARP2 by clinical PARP inhibitors. Cancer Res. 72, 5588-5599 (2012).

39. Nam, E. A. et al. Thr-1989 phosphorylation is a marker of active ataxia telangiectasia-mutated and Rad3-related (ATR) kinase. J. Biol. Chem. 286, 28707-28714 (2011).

40. Sartori, A. A. et al. Human CtIP promotes DNA end resection. Nature 450, 509-514 (2007).

41. Iacovoni, J. S. et al. High-resolution profiling of gammaH2AX around DNA double strand breaks in the mammalian genome. EMBO J. 29, 1446-1457 (2010).
42. Zhou, Y., Caron, P., Legube, G. \& Paull, T. T. Quantitation of DNA doublestrand break resection intermediates in human cells. Nucleic Acids Res. 42, e19 (2014).

43. Gallardo, I. F. et al. High-throughput universal DNA curtain arrays for singlemolecule fluorescence imaging. Langmuir 31, 10310-10317 (2015).

44. Krietsch, J. et al. PARP activation regulates the RNA-binding protein NONO in the DNA damage response to DNA double-strand breaks. Nucleic Acids Res. 40, 10287-10301 (2012).

45. Bunting et al. 53BP1 inhibits homologous recombination in Brcal-deficient cells by blocking resection of DNA breaks. Cell 141, 243-254 (2010).

46. Schultz, N., Lopez, E., Saleh-Gohari, N. \& Helleday, T. Poly(ADP-ribose) polymerase (PARP-1) has a controlling role in homologous recombination. Nucleic Acids Res. 31, 4959-4964 (2003).

47. He, Y. J. et al. DYNLL1 binds to MRE11 to limit DNA end resection in BRCA1-deficient cells. Nature 563, 522-526 (2018).

48. Noordermeer, S. M. et al. The shieldin complex mediates 53BP1-dependent DNA repair. Nature 560, 117-121 (2018).

49. Mirman, Z. et al. 53BP1-RIF1-shieldin counteracts DSB resection through CST- and Polalpha-dependent fill-in. Nature 560, 112-116 (2018).

50. Findlay, S. et al. SHLD2/FAM35A co-operates with REV7 to coordinate DNA double-strand break repair pathway choice. EMBO J. 37, e100158 (2018).

51. Spagnolo, L., Barbeau, J., Curtin, N. J., Morris, E. P. \& Pearl, L. H. Visualization of a DNA-PK/PARP1 complex. Nucleic Acids Res. 40, 4168-4177 (2012)

52. Wang, M. et al. PARP-1 and Ku compete for repair of DNA double strand breaks by distinct NHEJ pathways. Nucleic Acids Res. 34, 6170-6182 (2006)

53. Ying, S., Hamdy, F. C. \& Helleday, T. Mre11-dependent degradation of stalled DNA replication forks is prevented by BRCA2 and PARP1. Cancer Res. 72, 2814-2821 (2012)

54. Soni, A. et al. Inhibition of Parp1 by BMN673 effectively sensitizes cells to radiotherapy by upsetting the balance of repair pathways processing DNA double-strand breaks. Mol. Cancer Ther. 17, 2206-2216 (2018).

55. Fouquin, A. et al. PARP2 controls double-strand break repair pathway choice by limiting 53BP1 accumulation at DNA damage sites and promoting endresection. Nucleic Acids Res. 45, 12325-12339 (2017).

56. Shen, Y. et al. BMN 673, a novel and highly potent PARP1/2 inhibitor for the treatment of human cancers with DNA repair deficiency. Clin. Cancer Res. 19, 5003-5015 (2013)

57. Oikawa, A., Tohda, H., Kanai, M., Miwa, M. \& Sugimura, T. Inhibitors of poly (adenosine diphosphate ribose) polymerase induce sister chromatid exchanges. Biochem Biophys. Res. Commun. 97, 1311-1316 (1980)

58. Hori, T. High incidence of sister chromatid exchanges and chromatid interchanges in the conditions of lowered activity of poly(ADP-ribose) polymerase. Biochem Biophys. Res. Commun. 102, 38-45 (1981).

59. Morrison, C. et al. Genetic interaction between PARP and DNA-PK in V(D)J recombination and tumorigenesis. Nat. Genet. 17, 479-482 (1997)

60. $\mathrm{Hu}, \mathrm{Y}$. et al. PARP1-driven poly-ADP-ribosylation regulates BRCA1 function in homologous recombination-mediated DNA repair. Cancer Discov. 4, 1430-1447 (2014)

61. Gottipati, P. et al. Poly(ADP-ribose) polymerase is hyperactivated in homologous recombination-defective cells. Cancer Res. 70, 5389-5398 (2010).

62. Patel, A. G., Sarkaria, J. N. \& Kaufmann, S. H. Nonhomologous end joining drives poly(ADP-ribose) polymerase (PARP) inhibitor lethality in homologous recombination-deficient cells. Proc. Natl Acad. Sci. USA 108, 3406-3411 (2011).

63. Mirza, M. R. et al. Niraparib maintenance therapy in platinum-sensitive, recurrent ovarian cancer. New Engl. J. Med. 375, 2154-2164 (2016)

64. Zou, L., Cortez, D. \& Elledge, S. J. Regulation of ATR substrate selection by Rad17-dependent loading of Rad9 complexes onto chromatin. Genes Dev. 16 198-208 (2002)

65. Niraj, J. et al. The identification of FANCD2 DNA binding domains reveals nuclear localization sequences. Nucleic Acids Res. 45, 8341-8357 (2017).

66. Schonenberger, F., Deutzmann, A., Ferrando-May, E. \& Merhof, D. Discrimination of cell cycle phases in PCNA-immunolabeled cells. BMC Bioinformatics 16, 180 (2015).

67. Dery, U. et al. A glycine-arginine domain in control of the human MRE11 DNA repair protein. Mol. Cell. Biol. 28, 3058-3069 (2008).

68. Herbert, A. D., Carr, A. M. \& Hoffmann, E. FindFoci: a focus detection algorithm with automated parameter training that closely matches human assignments, reduces human inconsistencies and increases speed of analysis. PLoS ONE 9, e114749 (2014)

69. Langelier, M. F., Planck, J. L., Servent, K. M. \& Pascal, J. M. Purification of human PARP-1 and PARP-1 domains from Escherichia coli for structural and biochemical analysis. Methods Mol. Biol. 780, 209-226 (2011).

70. Buisson, R. et al. Cooperation of breast cancer proteins PALB2 and piccolo BRCA2 in stimulating homologous recombination. Nat. Struct. Mol. Biol. 17, 1247-1254 (2010). 
71. Yu, Z. et al. The MRE11 GAR motif regulates DNA double-strand break processing and ATR activation. Cell Res. 22, 305-320 (2012).

72. Henricksen, L. A., Umbricht, C. B. \& Wold, M. S. Recombinant replication protein A: expression, complex formation, and functional characterization. J. Biol. Chem. 269, 11121-11132 (1994).

73. Myler, L. R. et al. Single-molecule imaging reveals the mechanism of Exo1 regulation by single-stranded DNA binding proteins. Proc. Natl Acad. Sci. USA 113, E1170-E1179 (2016).

74. Efron, B. \& Tibshirani, R. An Introduction to the Bootstrap (Chapman \& Hall, London, 1993).

\section{Acknowledgements}

We would like to thank members of the Hendzel, Masson, and Poirier laboratories and Stéphane Richard, Michal Zimmerman, and Amélie Fradet-Turcotte for comments and suggestions. We also acknowledge Pablo Huertas for advice in performing SMART,

Hilmar Strickfaden for assistance in data quantification, Philipp Oberdoerffer (National Cancer Institute, USA) for the kind gift of TSI-DR-GFP U2OS reporter cells, Dr Gaëlle Legube (University of Toulouse, France) for the ER-AsiSI U2OS cell line, Roger Greenberg (University of Pennsylvania, USA) for U2OS cells stably expressing mCherryLacI-FokI construct, Scott Kern and Fumiko Esashi for DLD1 BRCA2+/- and DLD1 BRCA2 $-/-$ cells, and Jana Krietsch for generating the CRISPR HEK293T PARP-1 $(-I-)$ cells. L.R.M. is supported by the National Cancer Institute of the National Institutes of Health (F99CA212452). This research was supported by the National Institute of General Medical Sciences of the National Institutes of Health (R01GM120554 to I.J.F.); the National Cancer Institute of the National Institutes of Health (P01CA092584 to I.J.F.); CPRIT (R1214 to I.J.F.); the Welch Foundation (F-1808 to I.J.F.); the CIHR (BMA342854 to J.M.P.); and a CIHR grant to M.H., J.-Y.M., and G.P. (MOP311036). I.J.F. is a CPRIT Scholar in Cancer Research, J.O. was a recipient of a CRCHU de Quebec scholarship, and J.-Y.M. is a FRQS research chair in Genome Stability.

\section{Author contributions}

I.J.F., G.G.P., M.H., and J.-Y.M. conceived the studies. M.-C.C., A.S., L.R.M., J.O., M.T.F., A.R., Y.C., C.E., and J.-P.G. designed and carried out the experiments. M.-F.L. and J.M.P. provided purified proteins. M.H. and J.-Y.M. wrote the original draft and all authors edited the manuscript.

\section{Additional information}

Supplementary Information accompanies this paper at https://doi.org/10.1038/s41467019-10741-9.

Competing interests: The authors declare no competing interests.

Reprints and permission information is available online at http://npg.nature.com/ reprintsandpermissions/

Peer review information: Nature Communications thanks Michael Huen and other anonymous reviewer(s) for their contribution to the peer review of this work.

Publisher's note: Springer Nature remains neutral with regard to jurisdictional claims in published maps and institutional affiliations.

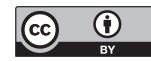

Open Access This article is licensed under a Creative Commons Attribution 4.0 International License, which permits use, sharing, adaptation, distribution and reproduction in any medium or format, as long as you give appropriate credit to the original author(s) and the source, provide a link to the Creative Commons license, and indicate if changes were made. The images or other third party material in this article are included in the article's Creative Commons license, unless indicated otherwise in a credit line to the material. If material is not included in the article's Creative Commons license and your intended use is not permitted by statutory regulation or exceeds the permitted use, you will need to obtain permission directly from the copyright holder. To view a copy of this license, visit http://creativecommons.org/ licenses/by/4.0/.

(C) The Author(s) 2019 